\title{
Dwelling on simple stimuli in visual search
}

\author{
Gernot Horstmann ${ }^{1}$. Stefanie I. Becker ${ }^{2}$. Anna Grubert ${ }^{3}$
}

Published online: 12 November 2019

(C) The Psychonomic Society, Inc. 2019

\begin{abstract}
Research and theories on visual search often focus on visual guidance to explain differences in search. Guidance is the tuning of attention to target features and facilitates search because distractors that do not show target features can be more effectively ignored (skipping). As a general rule, the better the guidance is, the more efficient search is. Correspondingly, behavioral experiments often interpreted differences in efficiency as reflecting varying degrees of attentional guidance. But other factors such as the time spent on processing a distractor (dwelling) or multiple visits to the same stimulus in a search display (revisiting) are also involved in determining search efficiency. While there is some research showing that dwelling and revisiting modulate search times in addition to skipping, the corresponding studies used complex naturalistic and category-defined stimuli. The present study tests whether results from prior research can be generalized to more simple stimuli, where target-distractor similarity, a strong factor influencing search performance, can be manipulated in a detailed fashion. Thus, in the present study, simple stimuli with varying degrees of target-distractor similarity were used to deliver conclusive evidence for the contribution of dwelling and revisiting to search performance. The results have theoretical and methodological implications: They imply that visual search models should not treat dwelling and revisiting as constants across varying levels of search efficiency and that behavioral search experiments are equivocal with respect to the responsible processing mechanisms underlying more versus less efficient search. We also suggest that eye-tracking methods may be used to disentangle different search components such as skipping, dwelling, and revisiting.
\end{abstract}

Keywords Attention: Selective · visual search · Eye movements and visual attention

\section{Introduction}

Finding a target among distractors can be easy or difficult, depending on the properties of the stimuli. It is easy in efficient search (also known as pop-out search), where the target is found at a glance, and where adding non-targets (distractors) to the search field does not affect search times. It is difficult in inefficient search, where a considerable amount of time is spent on checking distractors before the target is finally detected, so that search times are slower with more than with less distractors. According to Treisman's seminal Feature Integration Theory (FIT; Treisman, 1985;

Gernot Horstmann

gernot.horstmann@uni-bielefeld.de

1 Department of Psychology, Bielefeld University, PO Box 100 131, 33501 Bielefeld, Germany

2 University of Queensland, St Lucia, Australia

3 Durham University, Durham, UK
Treisman \& Gelade, 1980), efficient or pop-out search has been attributed to the use of the output of a parallel feature extraction stage, whereas inefficient search indicates the involvement of a capacity-limited attentional stage that operates at least partly in a serial manner. This concept has been elaborated on in more recent models of visual search, such as Guided Search (GS; Wolfe, Cave, \& Franzel, 1989; Wolfe, 1994, 2007), the Target Acquisition Model (TAM; e.g., Zelinsky, 2008), the Dimensional Weighting Model (DW; e.g., Found \& Müller, 1996), or the Saliency Model (SM; Itti \& Koch, 2000, 2001), all of which explain varying levels of search efficiency in particular by differences in target guidance. GS2 (Wolfe, 1994), for example, suggests that during an initial (bottom-up) stage of visual processing, the visual field is decomposed into isolated spatial maps of basic features for color, orientation, luminance, etc. The output of these separate maps is spatiotopically organized and summed up to be represented in a single spatiotopic map of activation. To enable guidance, searched-for features are boosted on the activation map. The profile of the activation map schedules sequential shifts of attention within the visual field towards 
conspicuous locations, the attentional visiting of which is necessary for the final decision whether a stimulus at a particular location is the target or not. A single high peak in the activation map leads to a rapid attention shift to the target's location, and search is efficient. However, if some of the distractors share features with the target, multiple peaks arise in the activation map. Because there is also inherent noise in the system, the target location may in this case not always have the highest activation peak on the map. Accordingly, multiple stimuli in the display, sometimes even all of them, may be visited before the target is finally found, rendering search inefficient. Note that this view predicts that search efficiency is a continuum rather than a dichotomy. Depending on the signal-to-noise ratio, search is predicted to be more or less efficient. This is reflected by the fact that search slopes, that is, the slopes of the functions relating reaction times to set size (i.e., the number of stimuli in a display), can vary between values around zero and several hundred milliseconds per item (Wolfe, 1998).

Guidance-based approaches to visual search are elegant and attractive not least because attentional guidance is effectively the only variable needed to explain search efficiency. These models predict that in efficient search, the ratio of activation for the target versus the distractors is very high, such that many of the distractors are not considered target candidates and will never be checked (skipping of distractors). Thus, prototypical efficient (pop-out) search is fast, because all distractors are skipped and only one stimulus, the target, is attended. Prototypical inefficient search is slow when all stimuli are considered target candidates, because attentionally investigating each of them is time consuming. Intermediate levels of efficiency result from possible but imperfect guidance by the target: The activation ratio for target against distractors is lower, and noise inherent in the activation map leads to the selection of some of the distractors in some of the trials. Hence, the target is not found as the first item, but it is also not found as a result of a random sampling of stimuli. Rather, a weak guidance signal renders search better than expected by chance. How much better, in turn, depends on the strength of the guidance signal.

On reflection, it is clear that other selection mechanisms should contribute to search difficulty (or its inverse: search efficiency) as well (Treisman \& Souther, 1985; Wolfe, 2001). The first of such mechanisms that we consider here is the time spent checking candidate target items or dwell time. It has been acknowledged that irrespective of whether a single stimulus (Wolfe et al., 1989; Zelinsky, 2008) or groups of stimuli (Hulleman \& Olivers, 2017) are attentionally investigated, the duration of this investigation process may be an effective cause rendering some searches inefficient, and that search is more efficient when only a short period of time is spent at the respective stimulus locations (e.g., Horstmann, Ansorge, \& Scharlau, 2006; Horstmann, Herwig, \& Becker, 2016; Horstmann, Becker, \& Ernst, 2017; Wolfe, 2001; Wolfe
\& Horowitz, 2017). Correspondingly, search is less efficient if, for whatever reasons, attentional checking time is increased in a particular type of search. In some sense this is also an obvious interpretation of search slopes, which is measured as the rise in milliseconds over added distractors to the search display: time spent checking each (additional) item. Note, however, that this is not the only interpretation of search slopes, because search slopes can also be interpreted as indicating the (average) number of distractors selected during search, which are selected with a constant search rate (e.g., Chun \& Wolfe, 1996).

The influence of attentional checking time on search time is no secret, and has been observed before (e.g., Hout et al., 2017; Walenchok et al., 2016; Wolfe, 2001; see Wolfe, 2018, for a comprehensive summary). However, guidancebased models tend to treat selection rate (e.g., Chun \& Wolfe, 1996), or dwell time (Zelinsky, 2008), as a constant that does not change for different search types. This does not necessarily mean that the processing requirements for stimuli are assumed to be the same for all variants of visual search. For example, Wolfe $(2003,2007)$ assumes parallel processing after selection (see also Venini et al., 2014) to reconcile the assumption of fast and constant selection rates in the range of $50 \mathrm{~ms} /$ item with findings that attentional dwell time is more in the range of 250-500 ms (e.g., Duncan et al., 1994). Yet current computational models of guided visual search, such as GS or TAM, fix this time to a constant (see, for a different treatment, the CRISP model by Nuthmann, Smith, Engbert \& Henderson, 2010, which is, however, not a model of guided visual search).

Treating attentional selection rate or dwell time as a constant implies that it is not useful in explaining differences in search. Moreover, yet another factor that might modulate search efficiency is the number of revisited stimuli in the visual field, i.e., stimuli that are checked multiple times. The classic notion in visual search models is that already visited locations are tagged in some way to ensure that each stimulus is investigated once, and ideally only once (although revisiting has been suggested previously, e.g., by Horowitz \& Wolfe, 1998). One possible way of implementing such tagging is inhibition of return (IOR; Posner, Rafal, Choate, \& Vaughan, 1985), where the locations on the activation map that correspond to already visited locations in the visual field are transiently suppressed (Itti \& Koch, 2000; Shipp, 2004; Wolfe et al., 1989; Zelinsky, 2008). Alternatively, already investigated locations in the visual field may be stored in a visuo-spatial memory store (i.e., VSTM; cf. Hulleman \& Olivers, 2017) and prevent revisiting via higher level processes concerned with choosing the target for the next fixation. Unless memory is perfect, capacity limitations and storage decay are responsible for revisiting already scanned locations and thus can explain a decrease of search efficiency. Capacity limitations, if relevant, should become more important as set 
size increases, while memory decay should become a determining factor for less efficient search with increasing time elapsed between stimulus onset and response.

Focusing on the single principle of guidance to explain many aspects of visual search performance adheres to the criterion of parsimony in theory building. Moreover, letting some factors vary while fixing others to constants is obviously wise when beginning to build computational models and explore their behavior through simulations and experiments. Scientific models often seek complexity reduction and include only a few important variables. One might even argue that dwelling (the time spent on checking a stimulus) and revisiting (the frequency of repeated checking) only affect search efficiency in such a minor way that it can hence be ignored. However, there is still a lot of variation in search efficiency in highly inefficient search, when guidance is difficult or absent, indicating that dwelling and revisiting may be important variables to explain visual search efficiency. In a series of previous studies we have used eye tracking to measure dwell time and revisiting most directly (Horstmann \& Becker, 2019; Horstmann, Ernst, \& Becker, 2019; Horstmann, Herwig, \& Becker, 2016; Horstmann, Becker, \& Ernst, 2017). Eye tracking was used to assess whether a distractor was looked at or not (skipping), and if so, for how long it was looked at during the first examination (dwelling) and whether the same distractor was looked at repeatedly (revisiting). Targets that were either similar or dissimilar to the distractors were used to induce different levels of search efficiency. We found that target-distractor similarity led to increased dwell time, which in turn increased search duration as indicated by response time (RT). Correspondingly, distractors were more often revisited when searching for a similar (difficult) target than when searching for a dissimilar (easy) target.

These previous studies used naturalistic face stimuli, which is a drawback for two reasons. First, visual search studies are usually conducted with well controlled laboratory stimuli. While the importance of using more ecologically relevant common stimulus categories (e.g., faces or scenes) in studies of visual search have been substantiated recently (e.g., Alexander \& Zelinsky, 2012; Einhäuser \& Nuthmann, 2016), research has revealed differences in the processing of artificial and realistic stimuli (e.g., Jenkins, Grubert, \& Eimer, 2018; Godwin, Walenchkok, Houpt, Hout, \& Goldinger, 2015; Neider \& Zelinsky, 2006; Zelinsky \& Schmidt, 2009). Second, while we successfully induced differences in search efficiency with different target categories in the previous studies, with natural stimuli it is not possible to actively manipulate theoretically important dimensions - in particular, target-distractors similarity (but see Hout et al., 2016, for procedures to measure target-distractor similarity).
The aim of the present study was to test whether our previous results for faces generalize to more standard laboratory stimuli, and to manipulate target-distractor similarity in a systematic way while using artificial stimuli. To that aim, we adapted search stimuli from an eye-tracking study by Reingold and Glaholt (2014), which seemed ideal for our paradigm as their study was already designed to manipulate target-distractor similarity. As illustrated in Fig. 1 (top panel), these stimuli were irregular shapes where the target differed from the distractors (bottom row) either only in the spatial organization of one individual stimulus part (similar targets where in comparison to the distractor stimuli, the left stimulus arm is shifted downwards along the attaching vertical bar; middle row), or - in addition - in the orientation of the entire configuration (dissimilar targets that are mirror versions of the similar targets; top row).

In Experiment 1, we produced ten variations (distortions) of the basic stimulus shapes by replacing some pixels of the stimulus figures with a different color (Fig. 1). This was done to mirror the stimulus heterogeneity of our previous studies where we used facial images of ten individuals (rather than using the same face ten times). Experiment 1 was therefore designed to allow for a direct comparison of potential effects caused by the stimulus material (heterogeneous faces vs. heterogeneous simple stimuli) rather than any other changes in the methods or materials. To test whether the artificially introduced distractor heterogeneity affected the results of Experiment 1, we conducted Experiment 2, in which we used the three original stimulus types without any distortions. In Experiment 3, we varied the set size to assess search efficiency in a more traditional way with RT increase as a function of increased stimulus number. Experiment 4 was designed to test whether the previously observed effects of not only skipping but also dwelling and revisiting would hold under search conditions that very likely promote strong guidance.

We expect target-distractor similarity to influence search times, both in target-present and target-absent trials. If the effect of similarity on search times is exclusively due to guidance, we would expect more skipping with dissimilar than similar targets, whereas dwelling and revisiting should be unaffected by similarity. More skipping in target-present trials would be because the dissimilar target has a higher probability of being selected early than the similar target. More skipping in target-absent trials would depend on a stopping rule that is correlated to the guidance in present trials (Chun \& Wolfe, 1996). In short, in target-absent trials, the activation map may contain multiple activations proportional to the similarity of the distractors with the searched for target, and, in addition, noise. Search is stopped when the remaining activation falls below some threshold, under which activation is treated as effectively zero. The threshold is set to an optimal value, just high enough so that the similar or dissimilar target, respectively, if present, is always above (note that this only applies to a 


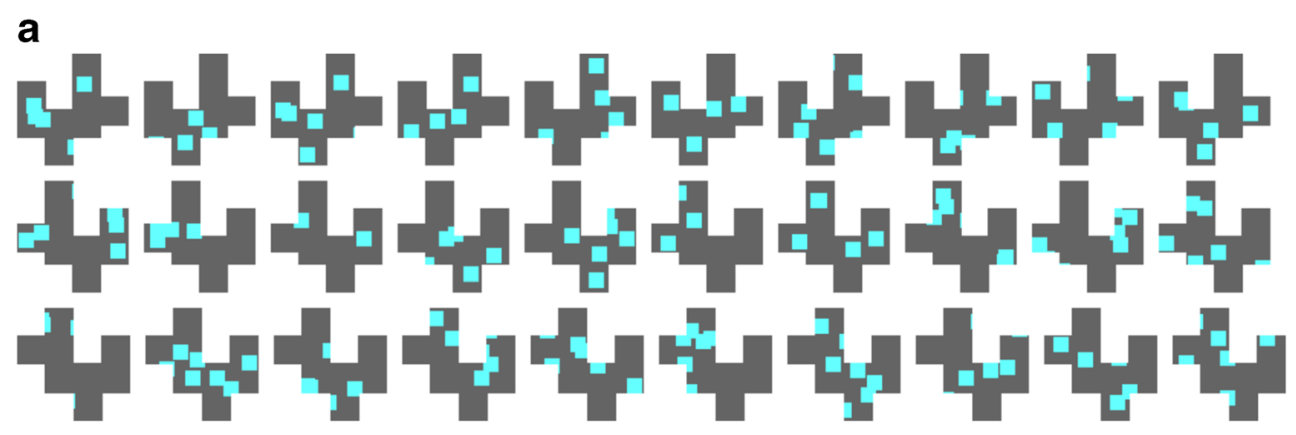

b

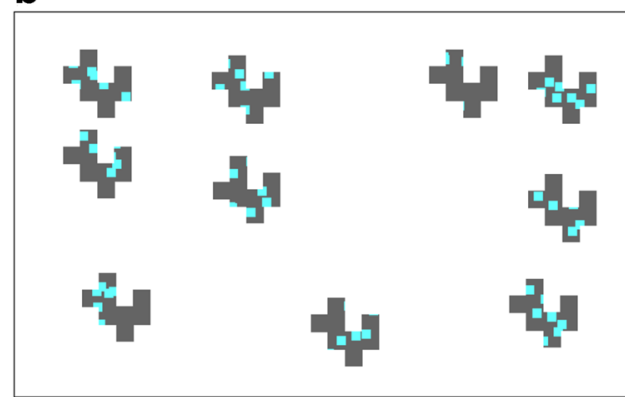

Fig. 1 A depicts the dissimilar targets (top row), the similar targets (middle row), and the distractors (bottom row), used in Experiment 1. B shows two example search displays with a similar (left panel) or dissimilar target (right panel), respectively. The right part includes a simplified scan path in yellow. The "lines" are saccades and the "knots" are fixations, where the eyes drift slowly on a small spot. The figure also illustrates the main variables. The time the gaze foveates a stimulus is the

design in which similar and dissimilar targets are presented in a blocked fashion). Because good guidance implies that the target has a much higher activation than a distractor, the threshold is well above zero in easy search. Because weak guidance implies that the target only statistically has a higher value than the distractors, the threshold is near zero in difficult search. In contrast, if the effect of similarity on search times is not exclusively due to guidance, but if dwelling and revisiting contribute to the effect of similarity on search times, we would expect to observe not only increased skipping rates, but also increased dwell times and revisiting rates when targetdistractor similarity is high.

To validate our hypotheses and predictions that not only skipping but also dwelling and revisiting are reliable determinants of search times, we traced the effects of these three variables by means of correlations and multiple regression analyses in which any potential impact will be registered in substantial regression weights. We focused on target-absent trials (cf. Horstmann et al., 2016, 2017, 2019) because they allow observations of distractor processing without interference from target-related processes. However, analyses for target-present trials are also reported for completeness. Note that it is not our aim to challenge guidance (here substantiated in skipping rates) as an important contributor to search efficiency in general. Rather, we aim to test whether differences in the selection mechanism that are not directly

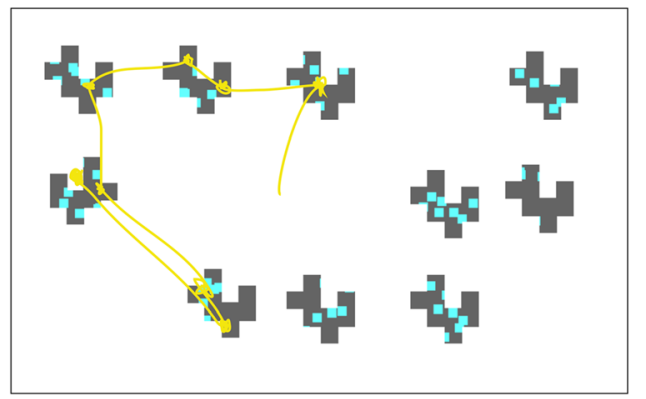

dwell time; this may include one fixation, as on the first stimulus visited, or two fixations, as for example on the second stimulus visited. The stimuli on the right side of the screen are not visited during this trial; that is the skipping proportion on this trial is 0.5 . The target is visited as the fourth stimulus, but it is revisited after fixating the fifth stimulus. As this is the only stimulus revisited on this trial, revising proportion for the target is 1.0 and revisiting proportion for the distractors is 0.0

related to guidance can modulate search efficiency in a non-trivial, substantial way.

\section{Experiment 1}

\section{Methods}

Participants Sixteen students with normal or corrected-tonormal vision participated in the study. Each received $€ 4$ for their 30-min participation. Three participants were excluded from further analysis because of near-chance performance in the similar target-present condition. The mean age of the remaining 13 participants was 23.3 years ( $\mathrm{SD}=2.3$ years); ten were female. The study was approved by Bielefeld University's ethics committee and performed in accordance with the approved guidelines.

Stimuli Figure 1A shows the stimuli used in Experiment 1. The basic shapes for the distractors (bottom row) and the similar targets (middle row; same as distractors, only that the right arm is shifted towards the bottom of the connecting vertical bar) were adapted from a publication of Reingold and Glaholt (2014). Their dissimilar targets, a C-shaped configuration, differed too much from the distractors for our purposes, and so we chose a horizontally flipped version of the similar 
target instead (top row). All stimuli were gray and were presented on a white background; each subtended $74 \times 74$ pixels. For Experiment 1, the basic shapes were distorted by randomly selecting seven positions within the inner $69 \times 69$ pixel matrix of a stimulus and flipping the gray foreground with blue. The selected locations to flip color each measured $11 \times$ 11 pixels, centered on the randomly selected position within the stimulus. No restrictions were applied to randomization (e.g., distortions were allowed to overlap), so that some of the distorted stimuli differed more from the basic shape than others.

Search displays consisted of ten stimuli presented at ten randomly selected locations from an imaginary grid of 5 horizontal $\times 3$ vertical locations. Figure $1 \mathrm{~B}$ shows two examples of target-present trials, one with a similar target (left panel) and one with a dissimilar target (right panel). The central position of the grid was excluded, as it contained the fixation marker in the pre-stimulus display. Center-to-center distances of the grid position were 100 pixels $\left(2.8^{\circ}\right.$ of visual angle) horizontally and 130 pixels vertically $\left(3.6^{\circ}\right)$. Each stimulus position on each trial was randomly jittered by $5 \pm 5$ pixels horizontally and vertically. The fixation marker was the standard fixation stimulus for the SR-1000 eye tracker (a black disk with a small white center).

Apparatus Stimuli were presented on a 19-in. display CRT monitor $(100-\mathrm{Hz}$ refresh rate, resolution $1,024 \times 768$ pixels) at a viewing distance of $71 \mathrm{~cm}$. A video-based tower-mounted eye tracker (EyeLink 1000, SR Research, Ontario, Canada) with a sampling rate of $1,000 \mathrm{~Hz}$ was used for the recording of eye movements. Participants' heads were stabilized by a chin and forehead rest, and for all participants the right eye was monitored. A 9-point eye-tracker calibration was used before the start of the experiment. Stimulus presentation and manual response collection was programmed using Experiment Builder 1.10.165 (SR Research, Ontario, Canada). Eyetracking data were preprocessed using Data Viewer 2.2.1 (SR Research, Ontario, Canada).

Design The experiment comprised six experimental blocks with 20 trials each. There were three alternating blocks for each of the two target categories - similar versus dissimilar targets. Half of the participants started with a similar target block. Each block contained ten target-present and ten target-absent trials. Target-absent trials displayed ten distractors, which meant that target-absent trials in the similar and dissimilar target conditions were structurally identical. In target-present trials, one of the distractors was randomly chosen to be replaced with one out of the ten possible target shapes of the respective target category (similar vs. dissimilar targets). The target in each trial was selected pseudo-randomly, so that each individual target shape appeared equally often in each block. Before the experiment proper participants completed a 20-trial practice block, which was not included in the analysis. The target category in this practice block was always different from the first experimental block.

Procedure Each trial started with a fixation control, which was terminated with a left-hand key press that initiated the presentation of the search display. Participants' task was to indicate with a right-hand (index or middle finger) key press whether or not one of the ten possible target shapes was present in the search display. The search display was shown until a manual response was registered. A short beep was issued in case of an error. Prior to each block, the ten possible targets of the respective target category (similar vs. dissimilar) and the ten possible distractors were displayed side by side until the participant initiated the start of the first trial. The experimental blocks were preceded by two practice blocks, one for each target type, which were not analyzed. Instructions emphasized speed and accuracy.

Data preprocessing Raw eye-position data were parsed by the eye-tracker software's standard experimental settings, which used a speed threshold $(30 \%)$ and an acceleration threshold $\left(8,000 \% \mathrm{~s}^{2}\right)$ for saccade detection. Rectangular $75 \times 75$ pixels areas of interest (AOIs) were defined that enclosed the stimulus shapes; outlier fixations were assigned to the nearest AOI. From these preprocessed data, four variables were derived for analysis. Each stimulus was classified as being fixated within a given trial or not. If a stimulus was fixated, dwell time was assessed, which is the sum of the fixation durations over the first continuous series of fixations on that stimulus. Of note, this measure often includes the duration of a single fixation, but in case several fixations were made on the stimulus, for example due to corrective saccades, the additional time was added. It must also be noted that only gaze duration during the first continuous visit was used here, dismissing the gaze duration of possible revisits. This was done to avoid confounding the measures of dwelling and revisiting. Furthermore, we recorded whether a stimulus was visited only once or whether it was revisited - that is, selected repeatedly during a trial after the first continuous run of fixations. A fixation was scored as a revisit if (a) the stimulus had been fixated before and (b) the last fixation of that stimulus was interrupted by at least one off-stimulus fixation. The basic variables of our analysis, however, were trial statistics (i.e., statistics for each trial). Skipping is defined as the proportion of stimuli that had not been fixated at all in a trial. Skipping is the variable that drives trial RTs, as assumed by guidance-based theories of visual search. Dwelling is the average dwell time in a given trial. We predict dwelling to be affected by similarity, whereas guidance-based theories treat dwelling as a constant. Also, we predict dwelling to substantially modulate trial RTs, i.e., to govern differences in search performance across trials. In addition to dwelling and skipping, we also assessed the 
proportion of stimuli that had been revisited, because Revisiting is a third possible source of variance in RTs. RT was measured as the time elapsed between display onset and key press of a correct answer.

Before analysis, raw data for the measures of time (i.e., correct RTs, dwell times, and stimulus selection latencies) were filtered for outliers. Measures of time were log transformed before outlier analysis. Data points were identified as outliers when they exceeded the mean of their respective condition (target presence $\times$ similarity) by two standard deviations or more. In addition, a lower cutoff was used for RTs (300 ms) and dwell times (40 ms). Outlier analysis was performed separately for each participant. As a result of the analysis, 36 RTs and 298 dwell times were removed.

\section{Results}

In the presentation of the results, our approach is as follows. First, we present error rates and manual RTs (Fig. 2, left column), along with the corresponding ANOVAs, to assess whether there was an effect of target-distractor similarity on overall search performance. Second, we show correlations and multiple regression analyses, where trial-based search times were regressed on the trial statistics for distractor dwelling, skipping, and revisiting, to indicate whether all these three underlying mechanisms are substantial predictors for increased search times (as indexed by RTs) in similar relative to dissimilar target search. The respective means (panels a, b, and c) are depicted in Fig. 3, top row. We do not present ANOVAs as we did in previous studies (e.g., Horstmann, Becker, \& Ernst, 2017) because the central evidence - that is the effects of similarity on skipping, revisiting, and, in particular, dwelling - is captured in the correlations between similarity on the one hand and skipping, revisiting, and dwelling on the other. Our main focus is on target-absent trials, because only in these trials can skipping, dwelling, and revisiting be observed independently of the processes that may lead to the selection of the target in target-present trials.

Error rates Mean proportion correct on target-absent trials was .99 and .98 in blocks with dissimilar and similar targets, respectively, and .94 and .89 on target-present trials in those blocks. A repeated-measures ANOVA with the factors target-distractor similarity (similar vs. dissimilar) and target presence (present vs. absent) revealed a significant main effect for presence, $F(1,12)=123.92, p<.001, \eta_{G}{ }^{2}=.48$, and targetdistractor similarity, $F(1,12)=6.94, p=.021, \eta_{G}^{2}=.20$, but no reliable interaction, $\mathrm{F}<1$. All trials with errors were excluded from further analyses.

Reaction times Figure 2 (left column) displays mean correct RTs - separately for target-absent and target-present trials in blocks with dissimilar and similar targets, respectively. A repeated-measures ANOVA computed over mean correct RTs with the factors target-distractor similarity (similar vs. dissimilar) and target presence (present vs. absent) revealed significantly shorter RTs on target-present than target-absent trials, $F(1,12)=168.56, \eta_{G}{ }^{2}=.38$, and in blocks with dissimilar as compared to similar targets, $F(1,12)=33.42, \eta_{G}^{2}=.31$; both $p \mathrm{~s}<.001$. The interaction just failed to be significant, $\mathrm{F}(1,12)=3.97, \mathrm{p}=.069$.

\section{Contributions of distractor dwelling, skipping, and revisiting to increased search times}

Search times are basically the product of the number of fixations and their average duration, and therefore it is reasonable to expect that skipping, dwelling, and revisiting together would predict search times very well. The crucial question is therefore not whether but to which degree these variables
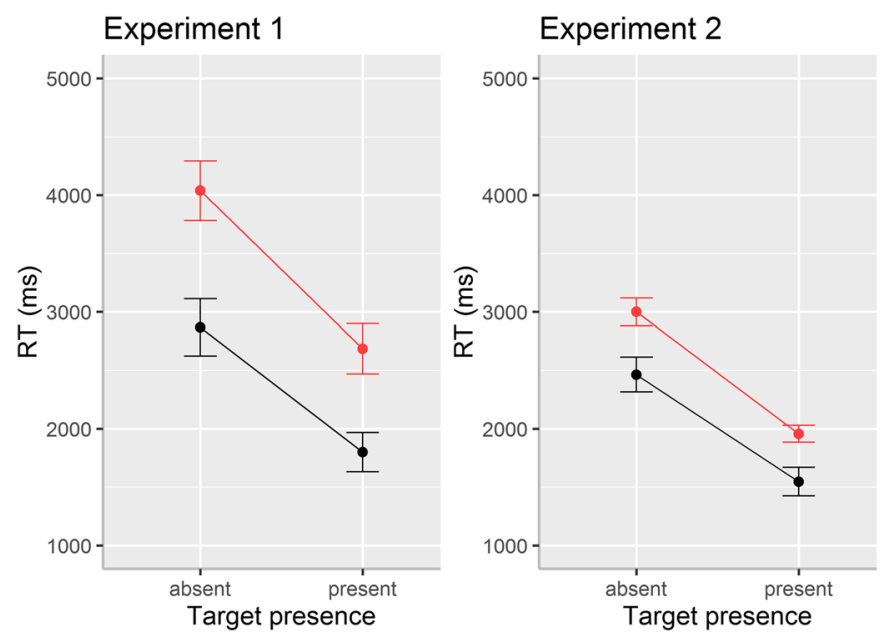

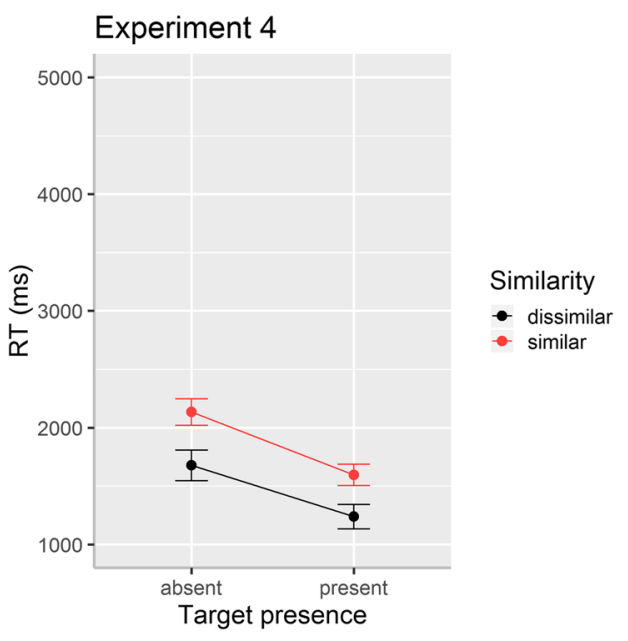

for Experiment 2 in the middle, and for Experiment 3 on the right. Error bars are standard errors (i.e., $\mathrm{SD} / \sqrt{ } \mathrm{N}$ ) of the means
Fig. 2 Mean correct response times (RTs) on target-absent and targetpresent trials for Experiments 1, 2, and 4, separately for blocks with dissimilar and similar targets. Results for Experiment 1 are on the left, 

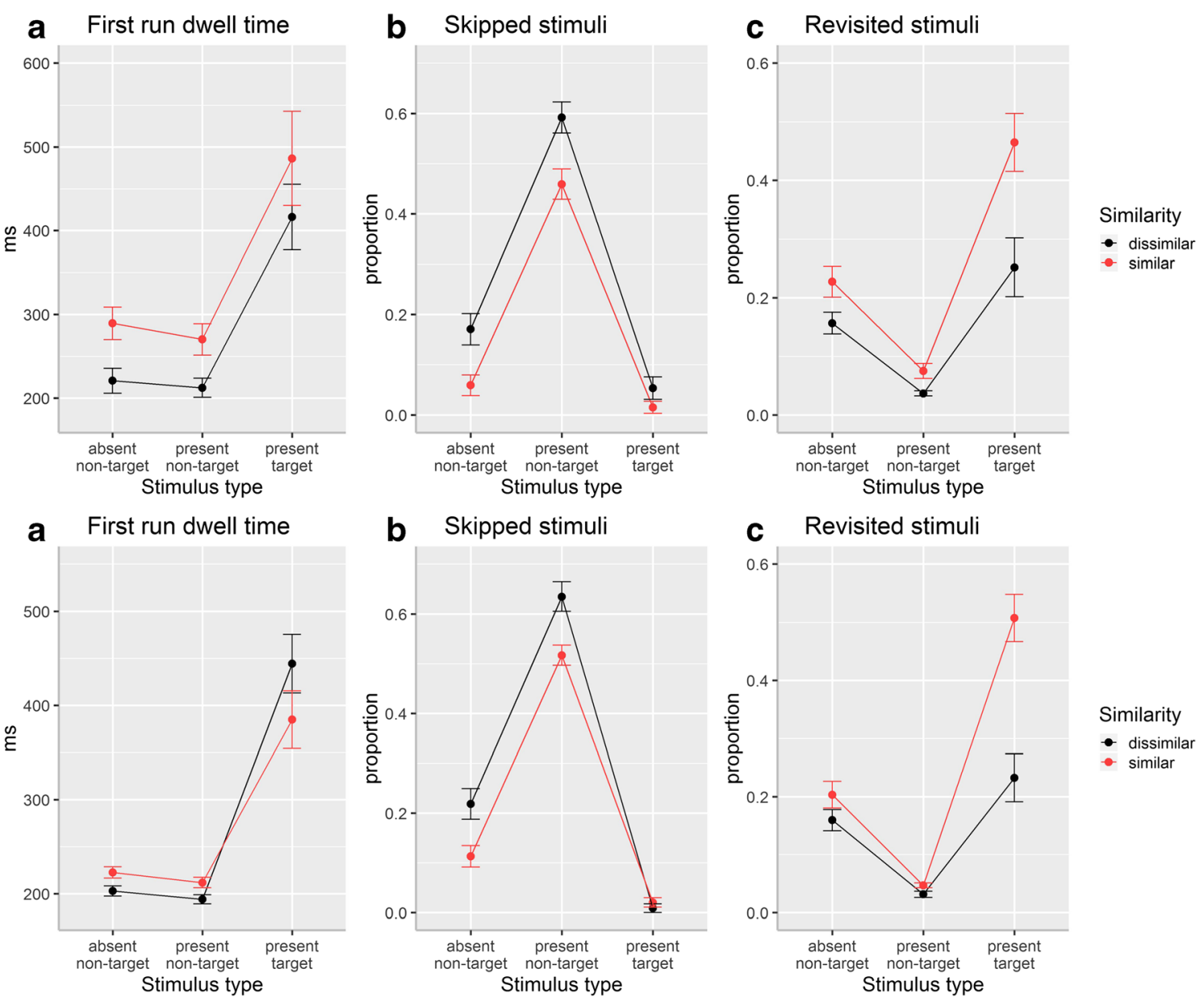

Fig. 3 Mean dwell times (a), proportion of skipped stimuli (b), and proportion of revisited stimuli (c) for distractors in target-absent trials, for distractors in target-present trials, and for targets in target-present

trials, separately for blocks with dissimilar and similar targets, respectively. Results for Experiment 1 are in the top row, and for Experiment 2 in the bottom row. Error bars are standard errors (i.e., $\mathrm{SD} / \sqrt{\mathrm{N}}$ ) of the means

predict RT. Table 1 (top panel) presents the bivariate correlations between manual RTs, the trial-based rates for revisiting and skipping, and the dwell times separately for target-absent and target-present trials. The unit of analysis is the trial, and each participant provides measures from up to 120 trials (minus the trials where outliers were detected or where the response was wrong). Note that the bivariate correlations (first column) between similarity on the one hand and RT, dwelling, skipping, and revisiting on the other hand correspond roughly to a test of the mean differences between the levels of similarity (i.e., the main effect of similarity), which would normally be done using a t-test. These significant correlations show that similarity impacts on RT, and also on dwelling, skipping, and revisiting.

The second column is informative, as it shows whether and how strongly dwelling, skipping, and revisiting co-vary with RTs. Figure 4 complements Table 1 with the respective scatterplots on target-absent trials separately for low (left panel) and high (right panel) target-distractor similarity trials. The fourth (bottom) row of Figure 4, for instance, shows the bivariate distributions of trial dwelling, trial skipping, trial revisiting, and trial RT for dissimilar (left) and similar (right) target blocks. Each dot represents one trial. For skipping and revisiting, random jitter was added to reduce overlap between the data points. The deep blue region represents the concentration of most trials. However, independently of the scatter, the positive linear regression slope of the main axis through the data points can clearly be visualized. Figure 4 also reveals that the linear relations between the variables are similar for blocks with similar and dissimilar targets. The same holds true for skipping and revisiting (middle and right plots of the fourth row). In contrast, dwelling showed no clear relationships to skipping (second row) and revisiting (third row, left plot), respectively. Finally, skipping and revisiting are slightly negatively correlated (third row, right plot), as the region above the diagonal is virtually devoid of data points. This has purely technical reasons and is due to the definition of revisiting as a per stimulus/trial statistic. Because a skipped distractor cannot 
Table 1 Correlation matrix for the variables target-distractor similarity, response times (RTs), skipping, dwelling, and revisiting in target-absent and target-present trials of Experiments 1 and 2

\begin{tabular}{lccll}
\hline & Similarity & RT & Skipping & Revisiting \\
\hline Experiment 1 & & & & \\
$\quad$ Target-absen trials & & & & \\
$\quad$ RT & .43 & & & \\
$\quad$ Skipping & -.40 & -.66 & & .04 \\
$\quad$ Revisiting & .19 & .49 & -.27 & \\
Dwelling & .43 & .80 & -.46 & \\
Target-present trials & & & & \\
RT & .34 & & & \\
Skipping & -.23 & -.81 & & \\
Revisiting & .28 & .51 & -.46 & \\
Dwelling & .21 & .23 & .11 & \\
Experiment 2 & & & & \\
Target-absent trials & & & & \\
RT & .34 & & & \\
Skipping & -.35 & -.74 & & \\
Revisiting & .12 & .69 & -.34 & \\
Dwelling & .28 & .55 & -.17 & \\
Target-present trials & & & & \\
RT & .26 & & .34 & \\
Skipping & -.22 & -.86 & & \\
Revisiting & .24 & .54 & -.45 & \\
Dwelling & -.12 & -.11 & .34 & \\
\hline
\end{tabular}

Note. Correlations were calculated on trial measures. All coefficients are significantly different from zero $(p<.05)$

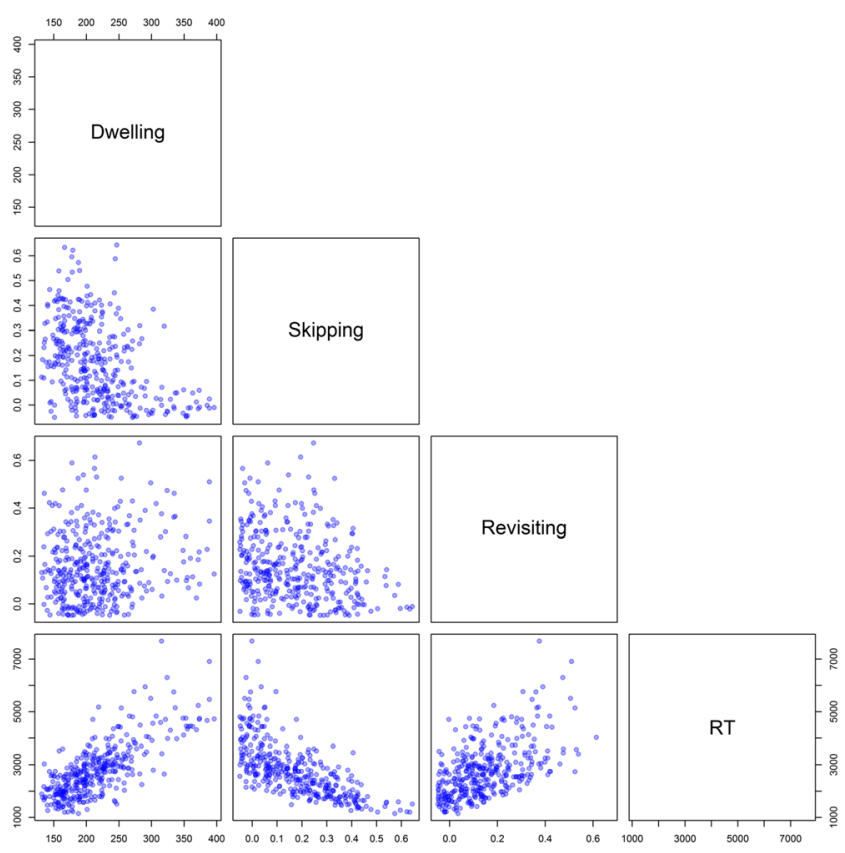

Fig. 4 Bivariate relationship between trial search times (RTs), revisiting rates (Revisiting), skipping rates (Skipping), and dwell times (Dwelling), for distractors in target-absent trials from Experiment 1, separately for the be revisited, as skipping rates go up revisiting rates go down. Note that due to the large number of observations even small coefficients are significantly different from zero. The correlations should thus be evaluated with respect to their size, not their statistical significance.

The correlations between RT and dwelling, skipping, and revisiting, respectively, cannot be easily interpreted because the predictors are themselves correlated. Accordingly, RT was regressed on dwelling, skipping, revisiting, and target-distractor similarity as predictors in order to obtain their statistically unique effects (i.e., when the other variables are statistically held constant). The binary variable target-distractor similarity was included to gauge the variance in RT that was caused by targetdistractor similarity, but was not transmitted to RT via our main predictor variables. This includes, among others, interactions between the predictor variables, which are not included in this simple linear model.

We used a linear multilevel regression with random intercepts to disentangle within-subject variations from betweensubject variations in dwelling, skipping, revisiting, and RT. Metric variables were $z$-transformed prior to analyses in order to make regression coefficients comparable among each other. With standardized coefficients, $b=0.5$ means that when the independent variable is increased by one standard deviation, the dependent variables increases for half a standard deviation. For the experimental factor of target-distractor similarity, dissimilar was coded as zero and similar as one. Regression coefficients are tested against zero with t-tests. We interpreted

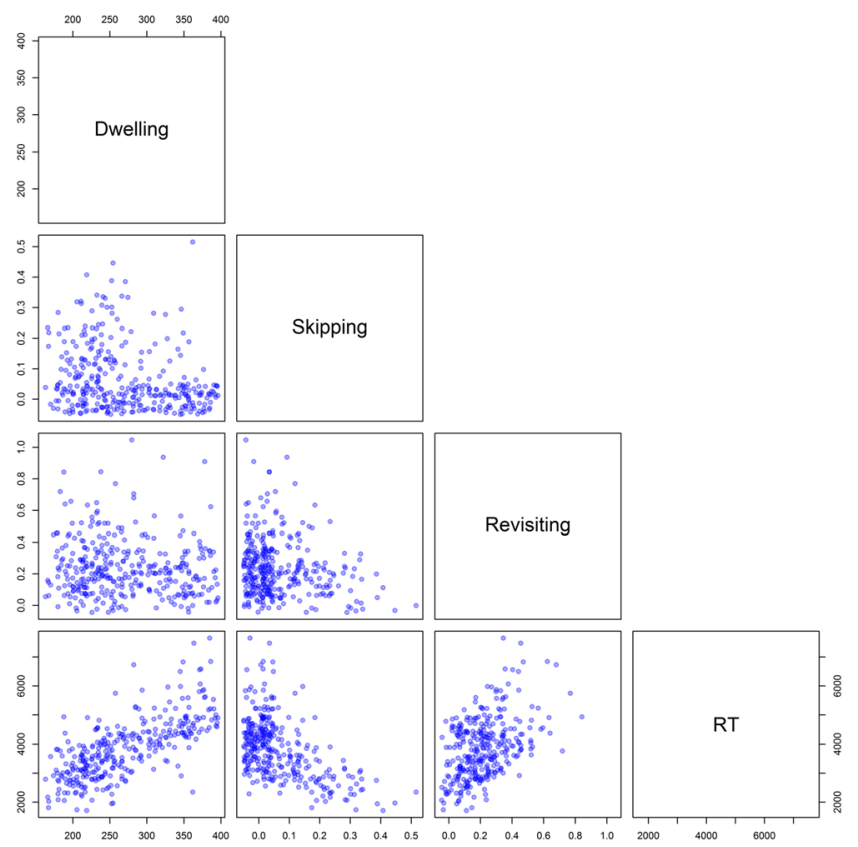

dissimilar target condition (left side) and the similar target condition (right side). Each dot represents a single trial 
empirical $t$-values exceeding a value of \pm 1.96 as significantly $(p<.05)$ differing from zero.

Target-absent trials Table 2 displays the results on targetabsent trials, based on 744 observations. The variance inflation factor (VIF) was used to guard against collinearity among the predictor variables. It was acceptable with tolerances (i.e., $1 / V I F$ ) within $>.70$ (well above the critical tolerance level of .10). All predictors (i.e., dwelling, skipping, revisiting) were found to have a significant effect on target-absent RTs. The effect of dwelling was strongest, followed by revisiting and then skipping (see the second column, where the regression slope, indicated by coefficient $\mathrm{b}$, is shown). Marginal $R^{2}$ was .90 (Nakagawa \& Schielzeth, 2013). The effect for targetdistractor similarity was significant but small, indicating that

Table 2 Linear multilevel regression of target-absent and target-present trial response times on dwelling, skipping, revisiting, and similarity as fixed effects, and random intercepts for participants based on the data of Experiments 1 and 2

Experiment 1

\begin{tabular}{|c|c|c|c|}
\hline Target-absent trials & $b$ & $S E(b)$ & $t$ \\
\hline Intercept & 0.10 & 0.04 & 2.65 \\
\hline Dwelling & 0.74 & 0.02 & 39.91 \\
\hline Skipping & -0.24 & 0.02 & -14.99 \\
\hline Revisiting & 0.44 & 0.01 & 30.68 \\
\hline Similarity & -0.10 & 0.03 & -3.59 \\
\hline \multicolumn{4}{|c|}{ Target-present trials } \\
\hline Intercept & -0.04 & 0.05 & -0.69 \\
\hline Dwelling & 0.25 & 0.02 & 11.92 \\
\hline Skipping & -0.73 & 0.02 & -37.71 \\
\hline Revisiting & 0.22 & 0.02 & 11.15 \\
\hline Similarity & 0.12 & 0.04 & 3.34 \\
\hline \multicolumn{4}{|l|}{ Experiment 2} \\
\hline \multicolumn{4}{|l|}{ Target-absent trials } \\
\hline Intercept & 0.06 & 0.02 & 2.87 \\
\hline Dwelling & 0.43 & 0.01 & 37.16 \\
\hline Skipping & -0.49 & 0.01 & -38.81 \\
\hline Revisiting & 0.52 & 0.01 & 43.54 \\
\hline Similarity & -0.01 & 0.02 & -0.49 \\
\hline \multicolumn{4}{|l|}{ Target-present trials } \\
\hline Intercept & 0.04 & 0.05 & 0.77 \\
\hline Dwelling & 0.20 & 0.02 & 11.61 \\
\hline Skipping & -0.84 & 0.02 & 44.75 \\
\hline Revisiting & 0.26 & 0.02 & 13.75 \\
\hline Similarity & 0.03 & 0.03 & 1.04 \\
\hline
\end{tabular}

$b$ regression coefficient; $S E$ standard error of regression coefficient

Models allowed for random intercepts between subjects; estimation method was full maximum likelihood; with the exception of similarity, all metric variables were $z$-transformed prior to analyses; for similarity "dissimilar target" was coded as zero and "similar target" as one Coefficients are statistically significant where $t>|1.96|$. target-distractor similarity had little unique influence on RTs after the common variance of dwelling, skipping, and revisiting had been removed.

Target-present trials Only distractor fixations were analyzed for target-present trials to be consistent with the previous analysis of target-absent trials. Table 2 shows the results on targetpresent trials, which were based on 701 observations. Indications of collinearity were low, with all computed tolerances $(1 / V I F)>.75$. All regression slopes were significant (see $t$-value). Skipping had the largest impact on target-present RTs, which is not surprising because the number of distractors that have been inspected before the target varies between zero and nine (all distractors in the display, ignoring possible revisits). Dwelling and revisiting had roughly the same beta weights, i.e., they can be considered to be equally important for the prediction of search RTs on target-present trials. Marginal $R^{2}$ was .79.

\section{Discussion}

Experiment 1 replicated previous findings (Horstmann, et al., 2016, 2017) and confirmed that in addition to skipping (the proportion of distractors that are excluded from inspection), dwelling (the time gaze is focused on a distractor) and revisiting (the frequency with which distractors are included for inspection repeatedly) were important predictors for search times. This is a notable finding because it shows that our previous observations are not limited to visual search for complex and naturalistic (face) stimuli. There are three main findings of Experiment 1. First, target distractor-similarity increases search times as expected, which is indicated by the significant correlation between similarity and RT, and the significant main effect of similarity in the ANOVA. The effect is strong, with search among ten items being almost a second longer with the similar target. Second, similarity increases dwelling and revisiting, and decreases skipping; these effects are also indicated by the corresponding correlation coefficients, and are visually presented in Figure 3, upper panel. These effects can be observed in absent and present trials as well. Third, dwelling, revisiting, and skipping, respectively, influence RT independently from each other, as indicated by the coefficients in the multiple regression. The effects of dwelling and revisiting are strong in absent trials, and are the strongest effects in this experiment. The effects of dwelling and revisiting are weaker in present trials, whereas the effect of skipping increases. We discuss this in more detail after we have reviewed the results of Experiment 2.

\section{Experiment 2}

Methodological aspects of stimulus presentation, experiment timing, and response collection were identical to Experiment 
1, with the important exception that in Experiment 2 we employed the undistorted prototypes of the stimuli used in Experiment 1 (i.e., the gray basic stimulus types presented in Fig. 1 without the colored distortions). The motivation for the stimulus distortions in Experiment 1 was to introduce a certain degree of stimulus variance (distractor-heterogeneity, cf. Duncan \& Humphreys, 1989) that was expected to approximate the differences in the stimulus material used by Horstmann et al. (2016, 2017), where target and distractor stimuli were images of ten individual faces, naturally introducing a certain level of stimulus variability. However, adding noise to the shapes may have had its own effect on the results. Moreover, the classic experiments concerned with the basic mechanisms of visual search often used little distractor heterogeneity (e.g., Treisman \& Souther, 1985; Wolfe, Cave, \& Franzel, 1989). Thus, Experiment 2 was designed to narrow the gap between more typical experiments on search efficiency and the protocols used in our studies. Apart from that, it is interesting in its own right to see whether the result pattern is changed when the distractors are more homogeneous (Duncan \& Humphreys, 1989). In general, we expect search to be more efficient with more homogeneous displays.

\section{Methods}

Participants Fourteen students participated in the study. Each received $€ 4$ for their 30-min participation. Two participants were excluded because of near-chance performance in the dissimilar target condition. Mean age of the remaining 12 participants was 26.4 years ( $\mathrm{SD}=2.8$ years); six were female. The study was approved by Bielefeld University's ethics committee and performed in accordance with the approved guidelines.

Apparatus, stimuli, and procedure All aspects of the stimulus parameters, the apparatus used for testing, the study design employed, the procedures applied, and eyetracking data preprocessing were identical to Experiment 1, with the only exception that in Experiment 2, stimuli were the undistorted prototype shapes. To reiterate the most relevant variables: Experiment 2 was tested in six blocks with 20 trials each, ten of which were targetpresent trials (search displays showed one target shape and nine distractor shapes), the other ten were targetabsent trials (search displays contained ten distractor shapes). Similar versus dissimilar targets were tested block-wise and alternatingly. Two practice blocks preceded the actual experimentation.

Data pre-processing This was analogous to Experiment 1 . Outlier detection led to the exclusion of 42 RTs and 274 dwell times.

\section{Results}

Error rates Mean proportion correct on target-absent trials was .99 and .96 in dissimilar and similar target blocks, respectively, and .95 and .87 on target-present trials of the respective blocks. A repeated-measures ANOVA with the factors targetdistractor similarity (similar vs. dissimilar) and target presence (present vs. absent) revealed significant main effects for target presence, $F(1,11)=19.81, p=.001, \eta_{G}{ }^{2}=0.29$, and targetdistractor similarity, $F(1,11)=10.05, p=.009, \eta_{G}{ }^{2}=0.19$, as well as a significant interaction $F(1,11)=5.21, p=.043, \eta_{G}{ }^{2}$ $=0.05$. While the mean proportion correct was at ceiling on target-absent trials, target-distractor similarity affected accuracy on target-present trials such that there were more errors in blocks with similar as compared to dissimilar targets.

Reaction times Figure 2, middle column, shows mean correct RTs, separately for target-absent and target-present trials in blocks with dissimilar and similar targets, respectively. Those mean correct RTs were subjected to a repeatedmeasures ANOVA with the factors target-distractor similarity (similar vs. dissimilar) and target presence (present vs. absent), which revealed significant main effects for target presence, $F(1,11)=134.83, p<.001, \eta_{G}{ }^{2}=0.60$, and targetdistractor similarity, $: F(1,11)=20.01, p=.001, \eta_{G}{ }^{2}=0.26$. The interaction was also significant, $F(1,11)=3.37, p=.093$ $\eta_{G}{ }^{2}=0.006$. RTs were faster on target-present than targetabsent trials (1,754 ms vs. $2,727 \mathrm{~ms})$, and in dissimilar than similar target blocks $(2,027 \mathrm{~ms}$ vs $2,454 \mathrm{~ms})$. As confirmed by two independent follow-up $t$-tests, the target-distractor similarity effect (i.e., slower RTs in similar relative to dissimilar target blocks) was more pronounced on target-absent trials, but was substantial on both target-present and target-absent trials, both $t(11)>3.79, p<.003$.

\section{Impact of similarity on dwelling, skipping, and revisiting, and contributions of distractor dwelling, skipping, and revisiting to search times}

As for Experiment 1, multiple regression analyses were trial based, and search times were regressed on the trial statistics for distractor dwelling, skipping, and revisiting, to investigate the predictive power of each of these mechanisms on search time. Figure 3, bottom panel, illustrates the respective means (panels $\mathrm{a}, \mathrm{b}$, and c). Table 11 presents the bivariate correlations between RTs and the rates for revisiting and skipping together with the dwell time, separately for target-absent and target-present trials. The first column of Table 1 (lower part) shows the correlations between similarity and RT, dwelling, skipping, and revisiting. All correlations were significantly different from zero, and the structure from Experiment 1 is well replicated. The second column shows the correlations 
between RT and dwelling, skipping, and revisiting with substantial effects as well. The two final columns show the correlations among dwelling, skipping, and revisiting.

Target-absent trials Table 2 (lower top panel) shows the statistical values obtained in the regression analyses based on 681 target-absent trials, reflecting the separate prediction power of dwelling, skipping, revisiting, and target-distractor similarity for the trial RTs. Collinearity among the predictor variables was low, with all $1 / V I F>.78$. All regression slopes except target-distractor similarity were significant. Marginal $R^{2}$ was .93 .

Target-present trials The lower bottom panel of Table 2 shows the respective values based on 632 target-present trials. Indications of collinearity were low, $1 / V I F>.75$. As on target-absent trials, all regression slopes were significant, except for the one on target-distractor similarity. Marginal $R^{2}$ was .82 .

\section{Discussion}

The most important result in Experiment 2 is that similarity has an effect on skipping, dwelling, and revisiting, and that these variables in turn have an effect on search times. This indicates that the general results pattern is observed independently from the presence (Experiment 1) or absence (Experiment 2) of distractor heterogeneity. The effects of similarity are generally somewhat weaker with homogeneous distractors, as indicated by the correlations between similarity and the measured variables (see Table 1, first column); however, all correlations are still solid. In the multiple regressions, the regression slopes of dwelling were reduced in comparison to Experiment 1, in particular for the target-absent trials; however, all regression slopes are still substantial. Overall, RTs seem to be shorter in Experiment 2 than in Experiment 1 (see Fig. 2), which is consistent with the assumption that distractorhomogeneity makes search easier. As the standard errors also happened to be different in the two experiments (see Fig. 2), it seems that increasing distractor homogeneity makes search less variable. To sum up, the present results show that dwelling and revisiting (together with skipping) significantly contributed to explaining the increased search RTs in similar versus dissimilar target blocks, irrespective of the lower distractor heterogeneity in Experiment 2.

\section{Experiment 3}

Experiment 3 included a variation of set size to test, with a more traditional approach - whether search for a dissimilar rather than a similar target is more efficient. For RT studies set size modulations are critical to separate the slope of the search function (indicator of the actual search mechanism) from the intercept (reflecting mainly response selection processes). One could argue that this would not be necessary in our experiments for which we employed eye movements to directly assess the search slopes. However, search slopes are a common currency in visual search and for the sake of between-study comparability it is thus informative to test search efficiency in this way. Furthermore, it might be possible that the impact of dwelling, skipping, and revisiting changes with set size. For example, revisiting may occur more frequently with higher set sizes because the decay of VSTM might be more relevant (Hulleman \& Olivers, 2017) or inhibition of return might be less effective with larger samples. We used ANOVAs to test whether dwelling, skipping, and revisiting are influenced by set size.

\section{Methods}

Participants Sixteen students participated in the study. Each received $€ 4$ for their 30 -min service. Two participants were excluded because of an excess of errors in at least one condition (33\% or more). Age from one participant was missing, mean age of the remaining participants was 26.15 years $(\mathrm{SD}=$ 1.91 years); nine were female, five were male. The study was approved by Bielefeld University's ethics committee and performed in accordance with the approved guidelines.

Apparatus, stimuli, and procedure All aspects of the stimulus parameters, the apparatus used, the study design employed, the procedures applied, and the eye-tracking data preprocessing were identical to Experiment 1 (with stimuli being randomly distorted), with two differences. First, set sizes of five and ten were used, both of which were presented intermixed within the same block of trials, doubling the number of trials per block to 40 . Second, stimuli were presented in a $5 \times 5$ grid, such that for both set sizes the arrangement of stimuli would be irregular on most of the trials.

Data pre-processing Data were preprocessed as in Experiments 1 and 2. Outlier screening led to the exclusion of 116 RTs, and 693 dwell times. As before, the screening was done case-wise and separately for each combination of the variables' similarity (similar vs. dissimilar), target presence (absent vs. present), and set size (5 vs. 10). Areas of interest (AOIs) for the eye-tracking data were defined somewhat differently from before, as every cell of the $5 \times 5$ grid was a single AOI. This was done because otherwise (i.e., using the nearest AOI for outlier fixations) AOI size would have been confounded with set size. 


\section{Results}

For Experiment 3, we report ANOVAs in addition to the correlations and the multiple regressions.

Error rates A repeated-measures ANOVA with the factors target-distractor similarity (similar vs. dissimilar), target presence (present vs. absent), and set size (5 vs. 10) revealed significant main effects for target presence, $F(1,13)=$ $30.3, p<.001, \eta_{G}{ }^{2}=0.36$, target-distractor similarity, $F(1,13)=21.93, p<.001, \eta_{G}{ }^{2}=0.18$, and set size, $F(1$, 13) $=5.11, p=.042, \eta_{G}{ }^{2}=0.02$. The Target Presence $\times$ Similarity interaction was significant, $F(1,13)=25.85, p$ $<.001, \eta_{G}{ }^{2}=0.20$, as was the Target Presence $\times$ Set size interaction, $F(1,13)=25.85, p<.001, \eta_{G}{ }^{2}=0.02$ (all other $\left.F_{\mathrm{S}}<1\right)$. Less errors were made in target-absent than target-present trials (.99 vs. .93), with dissimilar than similar targets (.98 vs. .94), and with higher than lower set size (.96 vs. 95). The Target Presence $\times$ Similarity interaction reflected larger absent-present difference in correct responses with high similarity (.99 vs. .89) than with low similarity (.99 vs. .97). The Target Presence $\times$ Set Size interaction was due to less errors with set size 5 than 10 in target-present trials (.94 vs. 92), whereas there were no set size differences in target-absent trials (.99 vs. .99).

Reaction times Figure 5 shows mean correct RTs. The same ANOVA as for error rates revealed all main effects and interactions to be significant for reaction times $\left(F_{\mathrm{S}}>6.11, p \mathrm{~s}<.028\right)$. To follow this up, we focused on search slopes of the RT-setsize function (difference in RT divided by difference in set

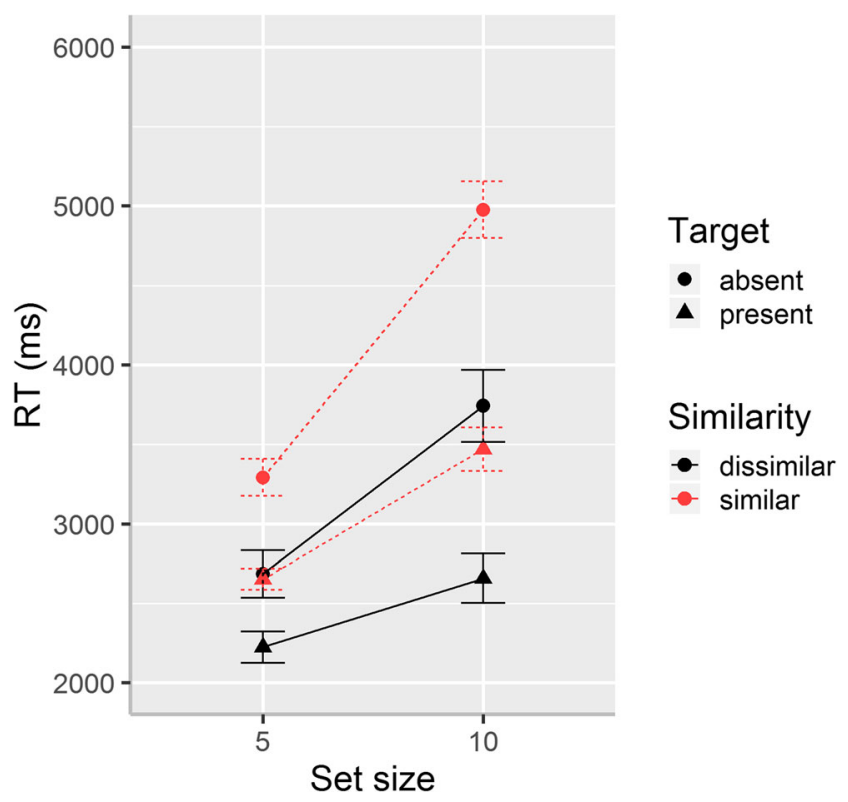

Fig. 5 Mean correct response times (RTs) for Experiment 3. Error bars are standard errors (i.e., $\mathrm{SD} / \sqrt{\mathrm{N}}$ ) of the means size). An ANOVA with the variables target presence and similarity revealed main effects for target presence, $F(1,13)=$ 137.01, $p<.001, \eta_{G}{ }^{2}=0.57$, and similarity, $F(1,13)=$ 42.96, $p<.001, \eta_{G}{ }^{2}=0.38$. The interaction was significant as well, $F(1,13)=6.11, p=.028, \eta_{G}{ }^{2}=0.03$. Search slopes were steeper in target-absent than target-present trials $(274 \mathrm{~ms} /$ item vs. $125 \mathrm{~ms} /$ item) and steeper with similar than dissimilar targets ( $250 \mathrm{~ms} /$ item vs. $149 \mathrm{~ms} /$ item). The difference between the target-absent and target-present slopes was larger with similar (164 ms/item vs. $337 \mathrm{~ms} /$ item) than with dissimilar targets ( $87 \mathrm{~ms} /$ item vs. $210 \mathrm{~ms} /$ item $)$.

Dwell times An ANOVA of the dwell times (Fig. 6a) with the variables target presence (present vs. absent), similarity (similar vs. dissimilar), and stimulus type (distractor in absent vs. distractor in present vs. target in present trial) rendered significant main effects for similarity, $F(1,13)=17.71, p=.001$, $\eta_{G}{ }^{2}=0.03$, and stimulus type, $F(2,26)=43.05, p<.001, \eta_{G}{ }^{2}=$ 0.57 , and set size, $F(1,13)=9.26, p=.009, \eta_{G}{ }^{2}=0.04$. Of the interactions, only the Stimulus Type $\times$ Set Size interaction was significant, $F(2,26)=5.13, p=.013, \eta_{G}{ }^{2}=0.04$ (other $F \mathrm{~s}<$ $2.77, p s>.080)$. The main effect for similarity was due to longer dwell times in blocks with similar than dissimilar targets (453 ms vs. 391). The main effect for stimulus type was in particular due to much longer dwell times on targets than distractors (see Fig. 6a); t-tests revealed, however that all stimulus types differed from each other, $t \mathrm{~s}>5.70, p s<.001$.

The interaction between set size and stimulus type was followed-up by three t-tests, each comparing similarity for each stimulus type. There was no significant effect of set size on dwelling on distractors in target-present trials (mean dwell time was $248 \mathrm{~ms}$ ). However, for distractors in target-absent trials, $t(13)=7.47, p<.001$, dwell time was longer with set size 5 than 10 (290 ms vs. $256 \mathrm{~ms}$ ), and for targets, dwell time was also longer with set size 5 than $10(640 \mathrm{~ms}$ vs. $516 \mathrm{~ms}), t(13)=2.51, p=.026$.

Proportion of fixated and skipped stimuli An ANOVA of the skipping proportions (Fig. 6b) with the variables target presence (present vs. absent), similarity (similar vs. dissimilar), and stimulus (distractor in absent vs. distractor in present vs. target in present trial) rendered significant results for all main effects and interactions, $F_{\mathrm{S}}>8.40, p_{\mathrm{S}}<.15$, with the exception of the similarity $\times$ set-size interaction, $F(1,13)=4.27, p=.06$.

Follow-up tests revealed that for distractors in targetabsent trials there was a significant main effect for similarity, $F(1,13)=48.34, p<.001, \eta_{G}{ }^{2}=0.54$, and for set size, $F(1,13)=84.0, p<.001, \eta_{G}{ }^{2}=0.40$. The two-way interaction was significant as well, $F(1,13)=31.51, p<$ $.001, \eta_{G}{ }^{2}=0.21$. Skipping was increased for dissimilar than similar targets (.10 vs. .02), and with set size 10 versus 5 (.09 vs. .03). This set size effect was larger for dissimilar than similar targets (difference .10 vs. .02). 

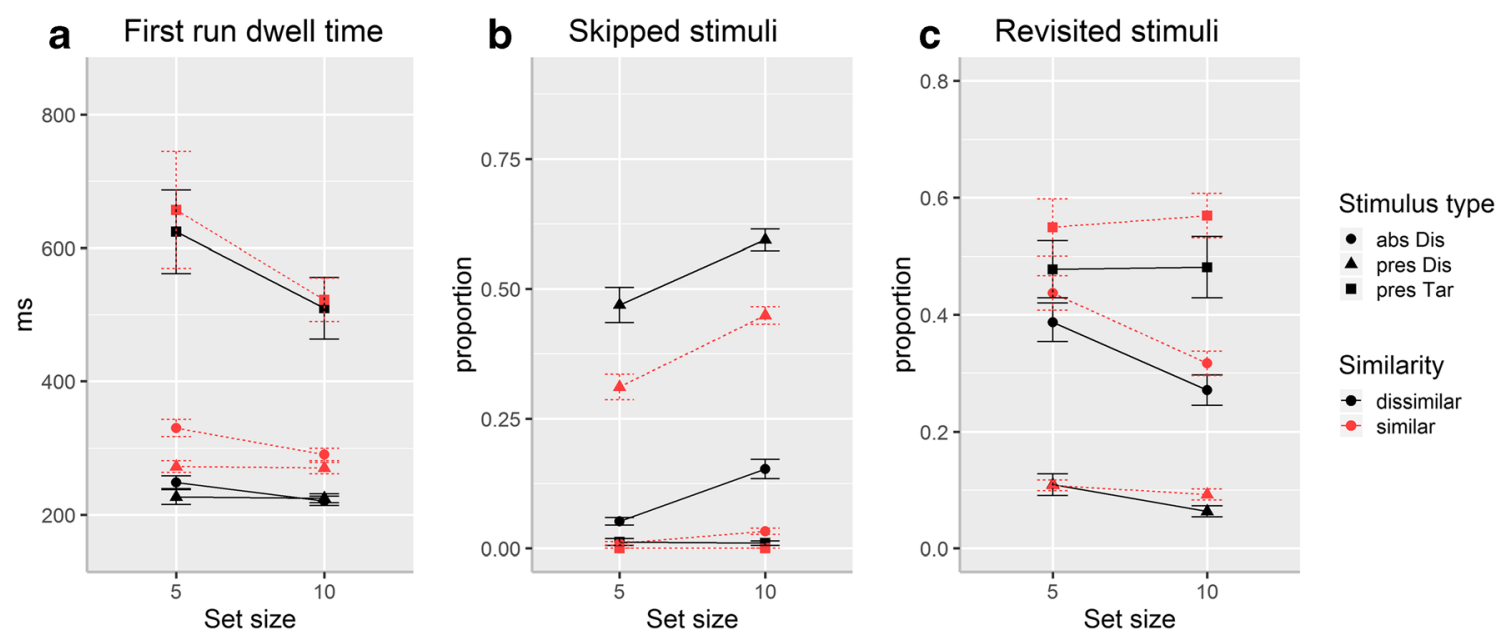

Fig. 6 Mean dwell times (a), proportion of skipped stimuli (b), and proportion of revisited stimuli (c) for Experiment 3. Error bars are standard errors (i.e., $\mathrm{SD} / \sqrt{ } \mathrm{N}$ ) of the means

A corresponding ANOVA for the distractors in targetpresent trials revealed main effects for similarity, $F(1,13)$ $=44.14, p<.001, \eta_{G}^{2}=0.42$, and set size, $F(1,13)=$ $67.30, p<.001 \eta_{G}{ }^{2}=0.35$. There was no reliable interaction, $\mathrm{F}<1$. More distractors were skipped with dissimilar than similar targets (.53 vs. .37), and when set size was 10 rather than 5 (.52 vs. .38).

Revisiting An ANOVA investigating the effects of set size on revisiting (Fig. 6c) revealed that revisiting is higher for similar than dissimilar targets $(.35$ vs. .30$), F(1,13)=5.53, p=.035$, $\eta_{G}^{2}=0.04$, higher for targets (.52) than for distractors in targetpresent (.09) and target-absent (.35) trials, $F(2,26)=93.57, p$ $<.001, \eta_{G}^{2}=0.69$, and higher for set size 5 than 10 (.34 vs. .30), $F(1,13)=15.56, p=.002, \eta_{G}^{2}=0.04$. Only the Stimulus Type $\times$ Set Size interaction reached significance, $F(2,26)=$ $23.37, p<.001, \eta_{G}^{2}=0.05$. The revisiting rate of the targets was virtually the same $(t<1)$ for set size 5 and $10(.51)$, but it was increased for distractors in target-present trials with set sizes 5 than 10 (.12 vs. .09), $t(13)=4.03, p<.002$, and for distractors in target-absent trials with set sizes 5 than $10(.41$ vs. .29), $t(15)=8.04, p<.001$.

\section{Impact of similarity on dwelling, skipping, and revisiting, and contributions of dwelling, skipping, and revisiting on search times}

Target-absent trials Correlations are shown in Table 3. As already indicated by the ANOVAs, the correlations of similarity with dwelling and skipping were substantial as before, the correlations of similarity and revisiting was significant but low here. The regression analyses corresponded to the previous analyses in Experiments 1 and 2, with the exception that set size was included as a factor. Also, interactions of set size with dwelling, skipping, and revisiting were now included to examine whether the effects of dwelling, skipping, and revisiting change across set sizes. Set size was dummy-coded as 0 for set size 5 and 1 for set size 10. There is no difference computationally between treating set size as a metric or categorical variable. The advantage of dummy-coding is that the simple regression coefficients can be read as showing the results for set size 5 , and the interactions coefficients give the increments for set size 10. As before, the metric predictors were standardized before regression analysis. In the following, we focus on the target-absent trials, as these are most informative.

Table 4 (top panel) shows the statistical values obtained in the regression analyses based on 1,596 target-absent trials. Collinearity among the predictor variables was stronger than in the previous experiments but still acceptable, with all $1 / V I F$

Table 3 Correlation matrix for the variables target-distractor similarity, set size, response time (RT), skipping, dwelling, and revisiting in targetabsent and target-present trials of Experiment 3

\begin{tabular}{|c|c|c|c|c|c|}
\hline & Similarity & Set size & RT & Skipping & Revisiting \\
\hline \multicolumn{6}{|c|}{ Target-absent trials } \\
\hline Set size & .00 & & & & \\
\hline RT & .38 & .57 & & & \\
\hline Skipping & -.40 & .31 & -.22 & & \\
\hline Revisiting & .11 & -.28 & .25 & -.31 & \\
\hline Dwelling & .54 & -.24 & .34 & -.28 & .20 \\
\hline \multicolumn{6}{|c|}{ Target-present trials } \\
\hline Set size & -.01 & & & & \\
\hline RT & .32 & .33 & & & \\
\hline Skipping & -.26 & .30 & -.54 & & \\
\hline Revisiting & .05 & -.13 & .34 & -.38 & \\
\hline Dwelling & .25 & -.02 & .25 & -.10 & .07 \\
\hline
\end{tabular}

Note. Correlations were calculated on trial measures. Coefficients $<|.07|$ are not statistically significant $(p<.05)$ 
Table 4 Linear multilevel regression of target-absent and target-present trial response times on dwelling, skipping, revisiting, similarity, and set size as fixed effects, and random intercepts for participants for Experiment 3

\begin{tabular}{|c|c|c|c|c|}
\hline \multirow[t]{10}{*}{ Target-absent trials } & & $\mathrm{b}$ & $\mathrm{SE}(\mathrm{b})$ & $\mathrm{t}$ \\
\hline & Intercept & -0.76 & 0.07 & -11.47 \\
\hline & Dwelling & 0.22 & 0.02 & 14.31 \\
\hline & Skipping & -0.10 & 0.02 & -5.73 \\
\hline & Revisiting & 0.20 & 0.01 & 14.54 \\
\hline & Similarity & 0.16 & 0.03 & 6.06 \\
\hline & Setsize & 1.62 & 0.02 & 73.18 \\
\hline & Dwelling:Setsize & 0.32 & 0.02 & 14.60 \\
\hline & Skipping:Setsize & -0.11 & 0.02 & -5.27 \\
\hline & Revisiting:Setsize & 0.29 & 0.02 & 12.92 \\
\hline \multicolumn{5}{|l|}{ Target-present trials } \\
\hline & Intercept & -0.53 & 0.08 & -6.74 \\
\hline & Dwelling & 0.06 & 0.02 & 3.99 \\
\hline & Skipping & -0.32 & 0.02 & -16.39 \\
\hline & Revisiting & 0.12 & 0.02 & 7.73 \\
\hline & Similarity & 0.26 & 0.03 & 10.37 \\
\hline & Setsize & 1.03 & 0.02 & 41.61 \\
\hline & Dwelling:Setsize & 0.12 & 0.02 & 4.75 \\
\hline & Skipping:Setsize & -0.56 & 0.03 & -19.35 \\
\hline & Revisiting:Setsize & 0.19 & 0.03 & 5.62 \\
\hline
\end{tabular}

$b$ regression coefficient; $S E$ standard error of regression coefficient

Models allowed for random intercepts between subjects; estimation method was full maximum likelihood; with the exceptions of similarity and set size, all metrical variables were $z$-transformed prior to analyses; for similarity, "dissimilar target" was coded as zero and "similar target" as one; for set size, "set size 5" was coded as zero and "set size 10" was coded as one

Coefficients are statistically significant where $t>|1.96|$

$>$.29. All predictors but similarity showed significant effects. Marginal $R^{2}$ was .78. Note that regression slopes for dwelling, skipping, and revisiting are for the set size 5 trials, and that the regression slopes for set size 10 can be derived from Table 4 by adding the interaction of respective predictor with set size. For instance, the regression slope for dwelling with set size 10 would be 0.54 , as regression coefficients are 0.22 for set size 5 and 0.32 for the increment with set size $10(0.22+0.32=0.54)$. The interactions of set size with dwelling skipping and revisiting, respectively, were significant. This indicates that the regression slopes differed significantly between the set sizes. More precisely, all regression slopes were more extreme in the set size 10 than in the set size 5 condition.

Target-present trials Table 4 (bottom panel) shows the results from the regression analyses based on 1,451 target-present trials. Collinearity was small, with all tolerances $(1 / V I F)>$ .42. All predictors had significant effects. Marginal $R^{2}$ was .70 .

\section{Discussion}

Experiment 3 yielded five informative results. First, not surprisingly, search was clearly inefficient, as gauged by the search slopes of the RT/set size function. Second, as predicted, search was less efficient for similar than dissimilar targets. Third, there was a small effect of set size on dwell time, with slightly prolonged dwell times at the smaller set size. The main reason for this may be that dwelling not only includes the time to analyze a currently fixated stimulus, but also the time to select the next fixation location, and to plan and prepare the corresponding saccade. With set size 5 , the spatial separation of the stimuli is necessarily higher in the present set up than with set size 10, and this might cause increased dwelling due to selection of a further-away location (which may be complicated by degraded visual acuity) and preparation of spatially longer saccades. There is as yet no consensus about which of the two variables is more affected by stimulus separations (Ludwig, Davies, \& Eckstein, 2014; Unema, Pannasch, Hoos, \& Velichkovsky, 2005; Antes, 1974, Viviani \& Swensson, 1982). Alternatively, it might be that with small set sizes, participants feel less pressured to work quickly on each individual item, because with few items, working somewhat slower does not affect search time much. Fourth, there was more skipping with set size 10 than 5, and this was more pronounced during search for dissimilar compared to similar targets, i.e., skipping increases in more densely populated displays. The observation of more skipping with larger set sizes is in accordance with the assumption that more than one stimulus can be processed within one fixation (e.g., Hulleman \& Olivers, 2017, Venini et al., 2014), given that the probability that an additional stimulus is in the functional field of view is lower with sparsely populated displays (e.g., in a set size 5 display). Finally, revisiting rates were higher with set size 5 than 10. This is a rather unexpected result, because revisiting has been tied to memory overflow (e.g., Hulleman \& Olivers, 2017) in that revisiting should occur more often for larger set sizes because participants forget the locations they already visited.

It is of note that the effects of dwelling, skipping, and revisiting in the regression analyses are remarkably stable, showing a similar pattern of effects as for Experiments 1 and 2: Dwelling and revisiting have both strong effects, and skipping a slightly weaker effect on search times in absent trials. All regression slopes were steeper with higher set sizes. This is not unexpected as dwelling, revisiting, and skipping are statistics per trial per stimulus. That is, an average dwell time of, for instance, $200 \mathrm{~ms}$ in a given trial means that each stimulus is looked at on average for $200 \mathrm{~ms}$. This in turn implies that dwelling would have a stronger impact on RT with more stimuli in the display. 


\section{Experiment 4}

Experiment 4 was designed to measure the effects of dwelling, skipping, and revisiting under conditions that most likely promote strong guidance (i.e., easy search). Given the inefficient searches employed in Experiments 1-3 (as indicated by the steep search slopes measured in Experiment 3, and the high search times in Experiments 1 and 2), it seems important to test whether dwelling, skipping, and revisiting still reliably contribute to search times when search might exclusively be controlled by guidance. In Experiment 4, we implemented guidance by allowing a subset search. Of the ten stimuli presented, five distractor stimuli had a color (orange) that was never the target color. The target, if present, was always among the stimuli with the other color (blue). Note that the coloring (blue vs. orange) uses a basic feature that is accessible before attentional deployment, and should thus be able to guide attention towards the subset of stimuli that possibly contains the target.

\section{Methods}

Participants Sixteen students participated in the study. Each received $€ 4-5$ for their 30- to 40-min service. Mean age (one age missing) was 25.21 years ( $\mathrm{SD}=3.24$ years); 13 were female.

Apparatus, stimuli, and procedure These were as in Experiment 1, with the exceptions that (1) the same $5 \times 5$ grid was used as in Experiment 3, (2) five of the distractors were shown in orange, while the other five stimuli (five distractors or four distractors and the target) were shown in blue, and (3) each block was twice as long and comprised 40 trials. Participants were informed about the target color (blue) at the beginning of the experiment and were therefore able to limit their search to five items in each trial.

Data pre-processing Data were preprocessed as in Experiments 1 and 2. Outlier screening led to the exclusion of 103 RTs, and 474 dwell times. As before, the screening was done case-wise and separately for each combination of the variables' similarity (similar vs. dissimilar), and target presence (absent vs. present).

\section{Results}

Error rates The ANOVA on error rates revealed only a main effect of similarity, $F(1,15)=11.29, p=.004, \eta_{G}{ }^{2}=0.11$ (other $F_{\mathrm{s}}<1$ ). More errors were made with similar than with dissimilar targets (.15 vs. .06).

Reaction times An ANOVA of the RTs with the variables target presence (present vs. absent) and similarity (similar vs. dissimilar) revealed main effects for target presence, $F(1$,
$15)=72.68, p<.001, \eta_{G}^{2}=0.25$, and similarity, $F(1,15)=$ $20.9, p<.001, \eta_{G}{ }^{2}=0.19$. The interaction was not significant, $F(1,15)=2.88, p=.111, \eta_{G}^{2}=0.003$. RTs were delayed in target-absent compared to target-present trials $(1,905 \mathrm{~ms}$ vs. $1,414 \mathrm{~ms}$ ), and they were slower with similar than with dissimilar targets $(1,456 \mathrm{~ms}$ vs. $1,864 \mathrm{~ms})$.

\section{Contributions of distractor dwelling, skipping, and revisiting to search times}

In contrast to the previous experiments, the search displays in Experiment 4 allow the participant to a priori exclude half of all display items in each trial (all orange items). This subset search is assumed to provide a strong incentive for participants to use guidance, which should consequently be reflected in increased skipping rates. Figure 7 shows the means (panels a, $\mathrm{b}$, and c) for dwelling, skipping, and revisiting for the factorial combinations of similar and dissimilar targets, and for distractors with or without the target color, for both targetabsent and target-present trials. Table 5 presents the bivariate correlations between RTs and the rates for revisiting and skipping together with the dwell time, separately for target-absent and target-present trials. The regression analyses corresponded in all details to those employed in the previous experiments.

Target-absent trials Table 6 (top panel) shows the results of the weights from the regression analyses based on 1,731 target-absent trials. Collinearity among the predictor variables was acceptable, with all $1 / V I F>$.73. All regression slopes were significant. The regression slopes of dwelling, skipping, and revisiting were of similar size. Marginal $R^{2}$ was .81 .

Target-present trials The bottom panel of Table 6 shows the respective values based on 1,589 target-present trials. Indications of collinearity were low, $1 / V I F>.88$. All regression slopes were significant. Marginal $R^{2}$ was .57 .

\section{Discussion}

Experiment 4 tested the replicability of the effects reported in the previous experiments under conditions when guidance is highly likely, due to the possibility to focus search on a subset of stimuli in each trial. As expected, search was relatively fast, because participants were able to ignore half of the distractors that did not share the color with the target (compare the RTs for Experiment 1 and Experiment 3 in Fig. 2). Also, skipping had a strong impact on search time in target-absent trials. Importantly, however, the weights for dwelling and revisiting were high as well, indicating that the presence of strongly guiding features does not eliminate the effects of dwelling and revisiting on search time. 

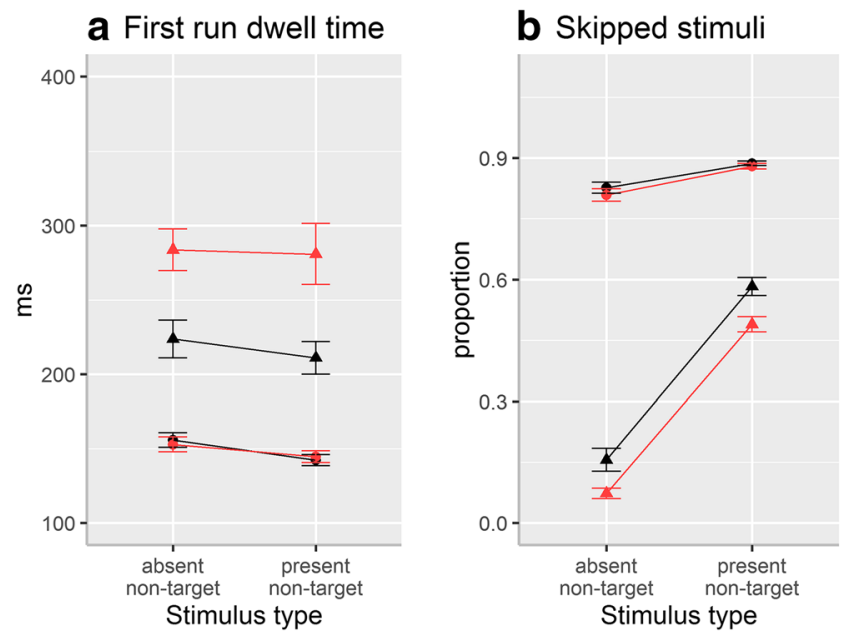

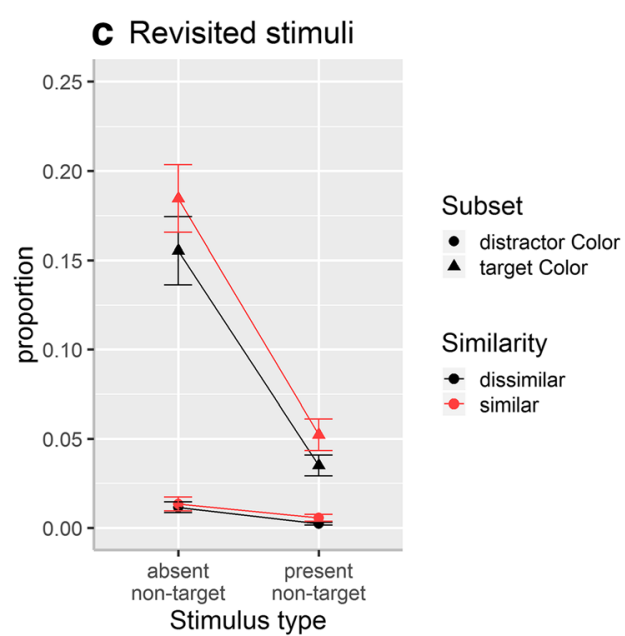

Fig. 7 Averages for Experiment 4. Mean dwell times (a), proportion of skipped stimuli (b), and proportion of revisited stimuli (c) for distractors, separately for similar and dissimilar targets, and for distractors with or without the basic feature characterizing the target. Error bars are standard errors (i.e., $\mathrm{SD} / \sqrt{ } \mathrm{N}$ ) of the means

classical findings of search slope modulations by targetdistractor similarity). Experiment 4 finally confirmed that the effects of skipping, dwelling, and revisiting persist even when search is more likely controlled by guidance.

We have pointed out that models of visual search often present elaborated theory on how guidance can be analyzed and how it affects search performance, and in particular search efficiency. Other aspects such as the contribution of attentional dwelling and revisiting are relatively underdeveloped,

Table 6 Linear multilevel regression of target-absent and target-present trial response times on dwelling, skipping, revisiting, and similarity as fixed effects, and random intercepts for participants based on the data of Experiment 4

Experiment 1

\begin{tabular}{clcc}
\hline Target-absent trials & $b$ & $S E(b)$ & $t$ \\
Intercept & 0.05 & 0.05 & $<|1|$ \\
Dwelling & 0.51 & 0.01 & 44.96 \\
Skipping & -0.40 & 0.01 & -38.75 \\
Revisiting & 0.40 & 0.01 & 39.99 \\
Similarity & 0.09 & 0.02 & 4.52 \\
Target-present trials & & & \\
Intercept & -0.08 & 0.09 & $<1 \mid$ \\
Dwelling & 0.28 & 0.01 & 18.93 \\
Skipping & -0.54 & 0.02 & -35.54 \\
Revisiting & 0.25 & 0.01 & 17.32 \\
Similarity & 0.20 & 0.03 & 7.44 \\
\hline
\end{tabular}

$b$ regression coefficient; $S E$ standard error of regression coefficient

Models allowed for random intercepts between subjects; estimation method was full maximum likelihood; with the exception of similarity, all metrical variables were $z$-transformed prior to analyses; for similarity "dissimilar target" was coded as zero and "similar target" as one Coefficients are statistically significant where $t>|1.96|$ 
which implies that their role in search is generally assumed to be relatively minor in nature. The present results, however, do not seem to support this presumption. In the present experiments, the effects of similarity on dwelling and revisiting are significant, as well as the effects of dwelling and revisiting on search times. Our results thus point to an explanatory gap in the contemporary theories of visual search and indicate that a successful search model should include the search mechanisms of dwelling and revisiting to explain more or less efficient search.

Experiment 3 revealed that search for the distorted version of the stimuli used in this study is very inefficient, with search slopes of $150 \mathrm{~ms} /$ item in target-present trials. Does this imply that the observed effects of dwelling and revisiting on search times are limited to inefficient searches where guidance (as reflected in skipping rates) is less likely to begin with? Experiment 4 tested this hypothesis and revealed that in target-absent trials (RT without contamination of any targetrelated processing), skipping rates were increased in Experiment 4 as compared to Experiment 1. This suggests that the sub-set search of Experiment 4 indeed allowed for a more guided search as compared to the full-set search employed in Experiment 1. Importantly, however, even under conditions that promote guidance, dwelling and revisiting still substantially contributed to the search RTs in Experiment 4. On a wider scale, our results suggest that guidance by target features (selection) as measured by increased skipping rates on the one hand and rejection of distractors as measured by decreased dwell times on the other hand are two mechanisms that coexist in visual search.

We would like to begin a closer inspection of our search variables with skipping. Skipping was influenced by similarity and in turn influenced search times, in all experiments, and in both target-present and -absent trials. Skipping in target-absent trials can be explained as a result of a variable threshold for search termination. In the context of GS (2.0; Wolfe, 1994), Chun and Wolfe (1996) showed that search is terminated when activation falls below the variable threshold. This threshold is set well above zero when target-distractor similarity is low (efficient search); however, with increased target-distractor similarity the threshold is set to a low value near zero. In consequence, search can be terminated early in the first case, but in the latter it may be that all stimuli of the display must be examined before search can be terminated. One might argue that skipping is not an exhaustive measure of "ignoring" a search item. Clearly, a peripheral stimulus can covertly be attended while focal attention is fixated at a different location in the visual field. Processing several items with a single fixation is also at the heart of the Functional View Field (FVF) model (Hulleman \& Olivers, 2017). The FVF is the region in space where a target can be detected among distractors with sufficient reliability in a single eye fixation. If the FVF includes more than one stimulus, this will result in the skipping of stimuli. The size of the FVF changes with search difficulty, and, thus, depending on whether more or less stimuli are included in the FVF, skipping rates may differ for similar and dissimilar targets. However, correlations between eye and attention movements are undoubtedly very high (Deubel \& Schneider, 1996), and we assume this to be sufficient to justify our methodological approach to employ fixations as an online marker of attentional selection (or de-selection, respectively).

In the context of skipping, we would like to state that despite the fact that we analyze and report search mechanisms in targetpresent trials, these results should be interpreted with caution. In particular when guidance is weak (inefficient search conditions), skipping rates will be dominated by random noise in the activation map. Finding the target as the first item (in which case the skipping rate for the distractors is 1.0 ) or as the tenth item (skipping rate of zero) has a very large effect on RT, and the high regression weights for skipping are therefore not surprising. However, the regression analyses on target-present trials uncovered significant weights for dwelling and revisiting as well, and therefore suggests a systematic contribution on search efficiency also on target-present and not just target-absent trials.

The second search-time predictor, dwelling, was increased by target-distractor similarity, and in turn increased RT. It is important to note that we may have overestimated dwell times due to two factors. First, eye-tracking procedures measure gaze fixations, which may be the sum of attentional dwell times on more than one stimulus included in one fixation (same logic as for skipping described above). Second, gaze dwelling does not only include the time of attentional dwelling, but also the time to select the next fixation target. While there is evidence that saccade planning is done in parallel to stimulus analysis (Ludwig, Davies, \& Eckstein, 2014), some studies report that saccade amplitude register in fixation duration, indicating that the processes are either not completely parallel or that saccade planning sometimes takes longer than stimulus analysis (Unema, Pannasch, Joos, \& Velichkovsky, 2005). This important caveat, in our assessment, does not invalidate gaze dwell time as a valid indicator of the duration of stimulus analysis. For example, Becker (2011) found that perceptual target difficulty (i.e., the acuity needed to detect the feature on which the response is based) increased dwell time independently from target-distractor similarity. While targetdistractor similarity theoretically influences both the categorization of a stimulus (attentional dwelling) and the selection of the next candidate stimulus, perceptual target difficulty should influence categorization exclusively. Moreover, in the difficult searches of Experiments 1-3, dwelling had substantial effects, but here it is not plausible that this effect was dominated by the selection processes: Apparently guidance was rather weak, because otherwise search would not have been this inefficient.

Finally, revisiting was assessed for two reasons. First, revisiting is an empirical fact, as for example described in IOR studies, and should thus be included in the equation that 
explains differences in search times. Second, revisiting has been brought into focus recently by the FVF model (Hulleman \& Olivers, 2016), which explicitly assumes that memory for recently checked positions is limited and that revisiting should thus be a general predictor of search behavior, in particular with larger set sizes. In line with these suggestions we find that revisiting is a substantial contributor to search performance. However, contrary to the suggestions in the FVF model, in Experiment 3, revisits were not more likely with the higher set size. Perhaps set size in our experiments was still too small (10) to tease out such effects of IOR and VSTM limitations? Of all variables tested in our experiments, revisiting was least affected by target-distractor similarity (see correlation Tables 1, 3, and 5). This corresponds to the idea that skipping is related to mechanisms such as IOR-decay time or VSTM capacity, which are independent on the actual search task.

For our study, we have used a relatively abstract "guidancebased model" as a reference frame, with GS, TAM, DW, or SM as possible exemplars. However, we acknowledge that this simplification does not do justice to the individual models, which do differ from each other in many respects. In particular, GS and DW are primarily attentional models, while TAM and SM are rather models of eye movements. Our results, which are based on eye movements, therefore relate more directly to TAM and SM. Conversely, for GS and DW, some of our underlying assumptions - as specified above (e.g., the interpretation of fixations as markers of attentional selection or the possible overestimation of attentional dwell time by empirical gaze dwell time) - may be more difficult to accept. GS (2.0; Wolfe, 1994) and TAM, while similar in many respects, are different in that GS treats near and far stimuli the same, while TAM employs the concept of an inhomogeneous retina, where stimuli closer to fixation are represented with higher visual acuity than more peripheral stimuli. According to GS, the activation map is thus computed once for each display (and updated with IOR for a visited stimulus), while TAM assumes a new coding of the activation map with every fixation, because every fixation changes the spatial parameters between the stimuli of a display. Irrespective of these important details, though, all of these models make important predictions for visual search performance. We suggest to complement these models with the search mechanisms dwelling, and revisiting, to accomodate the present finding that these mechanisms have a substantial impact on visual search performance.

On a broader scale, our results have important implications for the interpretation of behavioral search experiments that use RT to measure search performance. An idealized guidancebased model suggests that differences in search efficiency are solely caused by different guidance opportunities. More efficient search would be due to higher skipping rates, as efficient search allows for a priori rejections of distractors as possible target candidates. Correspondingly, less efficient search is due to low skipping rates, as multiple distractors have to be checked for target status. Because search efficiency is explained by guidance exclusively, it is tempting to interpret differences in search efficiency as directly indicating different degrees of guidance (given that other possible influences such as differential amounts of crowding can be excluded; cf. Vlaskamp \& Hooge, 2006). However, our series of experiments suggest that skipping is not the only mechanism determining visual search performance: dwelling and revisiting also significantly modulate search efficiency. The effects on dwelling and skipping, however, cannot be disentangled in RT data. RT measures, and in particular the measures of search efficiency derived from search function slopes, are completely blind as to whether these effects are driven by longer dwelling or less skipping or more frequent rescanning. Eye-tracking data, as used in the present experiments, are very useful to assess dwelling and skipping separately. At present, eye tracking seems to be the only method that allows distinguishing between skipping, dwelling, and revisiting.

Taken together, search slopes are a reliable measure of search efficiency, but they are not informative as to the underlying search mechanisms. Eye-tracking data are required to disentangle contributions of guidance/skipping, dwelling, and revisiting on search performance. Any comprehensive model of visual search must incorporate all three of those selection mechanisms: skipping, dwelling, and revisiting.

Funding This work was supported by the Cluster of Excellence Cognitive Interaction Technology 'CITEC' (EXC 277) at Bielefeld University, which is funded by the German Research Foundation (DFG), and by DFG grant HO 3248/2-1 to Gernot Horstmann.

\section{References}

Alexander, R. G., \& Zelinsky, G. J. (2012). Effects of part-based similarity on visual search: The Frankenbear experiment. Vision research, 54, 20-30.

Becker, S. I. (2011). Determinants of dwell time in visual search: Similarity or perceptual difficulty? PLOS ONE 6(3): e17740. doi https://doi.org/10.1371/journal.pone.0017740

Chun, M. M., \& Wolfe, J. M. (1996). Just say no: How are visual searches terminated when there is no target-present? Cognitive Psychology, 30, 39-78.

Deubel, H., \& Schneider, W. X. (1996). Saccade target selection and object recognition: Evidence for a common attentional mechanism. Vision Research, 36(12), 1827-1837.

Duncan, J., \& Humphreys, G. W. (1989). Visual search and stimulus similarity. Psychological Review, 96, 443-458.

Duncan, J., Ward, R., \& Shapiro, K. (1994). Direct measurement of attentional dwell time in human vision. Nature, 369(6478), 313-315.

Einhäuser, W., \& Nuthmann, A. (2016). Salient in space, salient in time: Fixation probability predicts fixation duration during natural scene viewing. Journal of Vision, 16(11): 13, 1-17.

Found, A., \& Müller, H. J. (1996). Searching for unknown feature targets on more than one dimension: Investigating a "dimensionweighting” account. Perception \& Psychophysics, 58(1), 88-101. 
Godwin, H. J., Reichle, E. D., \& Menneer, T. (2017). Modeling Lag-2 revisits to understand trade-offs in mixed control of fixation termination during visual search. Cognitive Science, 41(4), 996-1019.

Gould, J. D. (1967). Pattern-recognition and eye-movement parameters. Perception \& Psychophysics, 2, 399-407.

Hooge, I. T. C., \& Erkelens, C. J. (1998). Adjustment of fixation duration in visual search. Vision Research, 38, 1295-1302.

Horowitz, T. S., \& Wolfe, J. M. (1998). Visual search has no memory. Nature, 394, 575-577.

Horstmann, G., \& Becker, S. I. (2019). More efficient visual search for happy faces may not indicate guidance, but rather faster distractor rejection: Evidence from eye movements and fixations. Emotion. Advance online publication.

Horstmann, G., Scharlau, I., \& Ansorge, U. (2006). More efficient rejection of happy than of angry face distractors in visual search. Psychonomic Bulletin \& Review, 13(6), 1067-1073.

Horstmann, G., Lipp, O. V., \& Becker, S. I. (2012). Of toothy grins and angry snarls - Open mouth displays contribute to efficiency gains in search for emotional faces. Journal of Vision. 12 (5), 7.

Horstmann, G., Herwig, A., \& Becker, S. I. (2016). Distractor dwelling, skipping, and revisiting determine target-absent performance in difficult visual search. Frontiers in Psychology, 7, 1152.

Horstmann, G., Becker, S., \& Ernst, D. (2017). Dwelling, rescanning, and skipping of distractors explain search efficiency in difficult search better than guidance by the target. Visual Cognition, 25(1-3), 291-305.

Horstmann, G., Ernst, D., \& Becker, S.I. (2019). Dwelling on distractors varying in target-distractor similarity. Acta Psychologica, 198, in press.

Hout, M.C., Godwin, H.J., Fitzsimmons, G. Robbins, A., Menneer, T., \& Goldinger, S.D. (2016). Using multidimensional scaling to quantify similarity in visual search and beyond. Attention, Perception, \& Psychophysics, 78, 3-20.

Hout, M. C., Robbins, A., Godwin, H. J., Fitzsimmons, G, \& Scarince, C. (2017). Categorical templates are more useful when features are consistent: Evidence from eye-movements during search for societally important vehicles. Attention, Perception, \& Psychophysics, $79,1578-1592$

Hulleman, J. \& Olivers, C. N. L. (2016). The impending demise of the item in visual search. Behavioral and Brain Sciences, in press.

Hulleman, J., \& Olivers, C. N. (2017). The impending demise of the item in visual search. Behavioral and Brain Sciences, 40, 1-69.

Itti, L., \& Koch, C. (2001). Computational modelling of visual attention. Nature reviews neuroscience, 2(3), 194.

James R. Antes, (1974) The time course of picture viewing. Journal of Experimental Psychology 103 (1):62-70

Jenkins, M., Grubert, A., \& Eimer, M. (2018). Category-based attentional guidance can operate in parallel for multiple target objects. Biological Psychology, 135, 211-219.

Jeremy M. Wolfe, (2003) Moving towards solutions to some enduring controversies in visual search. Trends in Cognitive Sciences 7 (2):70-76

Laurent Itti, Christof Koch, (2000) A saliency-based search mechanism for overt and covert shifts of visual attention. Vision Research 40 (10-12):1489-1506

Ludwig, C. J., Davies, J. R., \& Eckstein, M. P. (2014). Foveal analysis and peripheral selection during active visual sampling. Proceedings of the National Academy of Sciences, 111(2), E291-E299.

Nakagawa, S., \& Schielzeth, H. (2013). A general and simple method for obtaining R2 from generalized linear mixed-effects models. Methods in Ecology and Evolution, 4(2), 133-142.

Neider, M. B., \& Zelinsky, G. J. (2006). Scene context guides eye movements during visual search. Vision Research, 46(5), 614-621.
Nuthmann, A., Smith, T. J., Engbert, R., \& Henderson, J. M. (2010). CRISP: a computational model of fixation durations in scene viewing. Psychological Review, 117(2), 382-405.

Posner, M. I., Rafal, R. D., Choate, L. S., \& Vaughan, J. (1985). Inhibition of return: Neural basis and function. Cognitive Neuropsychology, 2(3), 211-228.

Reingold, E. M., \& Glaholt, M. G. (2014). Cognitive control of fixation duration in visual search: The role of extrafoveal processing. Visual Cognition, 22(3-4), 610-634.

Shipp, S. (2004). The brain circuitry of attention. Trends in Cognitive Sciences, 8(5), 223-230.

Treisman, A. (1985). Preattentive processing in vision. Computer vision, graphics, and image processing, 31(2), 156-177.

Treisman, A. M., \& Gelade, G. (1980). A feature-integration theory of attention. Cognitive Psychology, 12, 97-136.

Treisman, A., \& Souther, J. (1985). Search asymmetry: A diagnostic for preattentive processing of separable features. Journal of Experimental Psychology: General, 114(3), 285-310.

Unema, P. J., Pannasch, S., Joos, M., \& Velichkovsky, B. M. (2005). Time course of information processing during scene perception: The relationship between saccade amplitude and fixation duration. Visual Cognition, 12(3), 473-494.

Venini, D., Remington, R.W., Horstmann, G., \& Becker, S.I. (2014). Centre-of-gravity fixations in visual search: When looking at nothing helps to find something. Journal of Ophthalmology, 237812, 1-14.

Viviani, P., \& Swensson, R. G. (1982). Saccadic eye movements to peripherally discriminated visual targets. Journal of Experimental Psychology: Human Perception and Performance, 8(1), 113-126.

Vlaskamp, B. N., \& Hooge, I. T. C. (2006). Crowding degrades saccadic search performance. Vision Research, 46(3), 417-425.

Walenchok, S. C., Hout, M. C., \& Goldinger, S. D. (2016). Implicit object naming in visual search: Evidence from phonological competition. Attention, Perception, \& Psychophysics, 78, 2633-2654

Wolfe, J. M. (1994). Guided Search 2.0: A revised model of guided search. Psychonomic Bulletin \& Review, 1, 202-238.

Wolfe, J. M. (1998). What can 1 million trials tell us about visual search? Psychological Science, 9, 33-39.

Wolfe, J. M. (2001). Asymmetries in visual search: An Introduction. Perception and Psychophysics, 63(3), 381-389.

Wolfe J. M. (2007). Guided search 4.0: Current progress with a model of visual search. In W. Gray (Ed.), Integrated models of cognitive systems (pp. 99-119). New York: Oxford.

Wolfe, J. M. (2018). Visual Search. In J. Wixted) (Ed.), Stevens' Handbook of Experimental Psychology and Cognitive Neuroscience (Vol. II. Sensation, Perception \& Attention: John Serences (UCSD)): Wiley.

Wolfe, J. M., \& Horowitz, T. S. (2017). Five factors that guide attention in visual search. Nature Human Behaviour, 1(3), 0058.

Wolfe, J. M., Cave, K. R., \& Franzel, S. L. (1989). Guided search: An alternative to the feature integration model for visual search. Journal of Experimental Psychology: Human Perception and Performance, 15(3), 419.

Zelinsky, G. J. (2008). A theory of eye movements during target acquisition. Psychological Review, 115, 787-835.

Zelinsky, G. J., \& Schmidt, J. (2009). An effect of referential scene constraint on search implies scene segmentation. Visual Cognition, 17(6), 1004-1028.

Publisher's note Springer Nature remains neutral with regard to jurisdictional claims in published maps and institutional affiliations. 